\title{
Integrated aircraft-path assignment and robust schedule design with cruise speed control
}

\author{
Özge Şafak ${ }^{\mathrm{a}}$, Siñan Gürel ${ }^{\mathrm{b}}$, M. Selim Aktürk ${ }^{\mathrm{a}, *}$ \\ a Department of Industrial Engineering, Bilkent University, 06800 Bilkent, Ankara, Turkey \\ ${ }^{\mathrm{b}}$ Department of Industrial Engineering, Middle East Technical University, Ankara 06800, Turkey
}

\section{A R T I C L E I N F O}

\section{Article history:}

Received 25 October 2015

Revised 11 March 2017

Accepted 12 March 2017

Available online 22 March 2017

\section{Keywords:}

Fleet type assignment

Airline scheduling

Cruise time controllability

Second order conic programming

Chance constraints

\begin{abstract}
A B S T R A C T
Assignment of aircraft types, each having different seat capacity, operational expenses and availabilities, critically affects airlines' overall cost. In this paper, we assign fleet types to paths by considering not only flight timing and passenger demand, as commonly done in the literature, but also operational expenses, such as fuel burn and carbon emission costs associated with adjusting the cruise speed to ensure the passenger connections. In response to flight time uncertainty due to the airport congestions, we allow minor adjustments on the flight departure times in addition to cruise speed control, thereby satisfying the passenger connections at a desired service level. We model the uncertainty in flight duration via a random variable arising in chance constraints to ensure the passenger connections. Nonlinear fuel and carbon emission cost functions, chance constraints and binary aircraft assignment decisions make the problem significantly more difficult. To handle them, we use mixed-integer second order cone programming. We compare the performance of a schedule generated by the proposed model to the published schedule for a major U.S. airline. On the average, there exists a $20 \%$ overall operational cost saving compared to the published schedule. To solve the large scale problems in a reasonable time, we also develop a two-stage algorithm, which decomposes the problem into planning stages such as aircraft-path assignment and robust schedule generation, and then solves them sequentially.
\end{abstract}

(c) 2017 Elsevier Ltd. All rights reserved.

\section{Introduction}

To achieve significant cost savings for the airline industry, the fuel cost, which has the major component of overall operational expenses, should be considered in the airline scheduling process. In general, fuel expenses account for about $30 \%$ of total operating cost. However, in 2008, the share for fuel costs rose to $50 \%$ with the sharp increase in the fuel prices (ICAO, 2009). In addition to rising fuel costs, greenhouse gas emissions are becoming significantly more important for airlines, as climate change is becoming an ever more important subject in the world. Aviation was the first sector to agree upon ambitious targets. One of the targets is a $50 \%$ reduction in net aviation $\mathrm{CO}_{2}$ emissions by 2050 compared to 2005 levels (IATA, 2013). Airlines have committed to achieve these through improved engine technologies, infrastructure improvement and better operations. As discussed in Marais and Waitz (2009), fuel consumption per passenger kilometer has decreased by $70 \%$ over the past four decades. In response to fuel expenses and emission restrictions, in this paper, we manage airline operations effi-

\footnotetext{
* Corresponding author.

E-mail address: akturk@bilkent.edu.tr (M.S. Aktürk).
}

ciently, such as flight re-timing and fleet assignment to minimize the fuel burn and $\mathrm{CO}_{2}$ emission costs by incorporating cruise speed control to ensure passenger connections.

Assignment of aircraft types, each having different seat capacity, operational expenses and availabilities, has a significant impact on the airlines' overall cost. In this paper, we assign fleet types by considering not only flight timing and passenger demand, as commonly done in the literature, but also operational expenses, such as fuel burn and carbon emission costs. We generate a robust schedule with improved capability in response to flight time uncertainty due to airport congestions. We simultaneously re-time the flight departure times and control the cruise speed to ensure the passenger connections at a desired service level. We provide more slack over vulnerable connections at the congested airports and remove excess slack from the remaining connections. We can increase the connection possibilities by increasing the cruise speed and inserting idle times. However, this strategy incurs additional cost of idle times and costs of fuel and carbon emissions associated with speed adjustment. An important question arises as to whether one could improve the solution through assigning a fuel efficient aircraft to an aircraft path with a high variability, which also has connected passengers. If a fuel efficient but smaller aircraft is assigned to 
a flight, some of the passengers may not be accommodated due to the limited seat capacity and be turned away by the airline, thus resulting in high passenger spill costs. If a larger aircraft with higher seat capacity is assigned to the same flight, passenger demand could be met but this directly increases the flight operating costs such as fuel and emission costs. Therefore, there is a need to consider all of these interrelated cost terms simultaneously to minimize the overall operating cost of the entire flight network.

Hai and Barnhart (2013) has introduced a dynamic airline scheduling approach that changes the schedule slightly by retiming and re-fleeting during the booking period. To reflect changing demand, re-timing allows the flight departure times slightly vary within specified time intervals to increase the passenger connection opportunities and a more cost effective fleeting possibility. Based on the updated passenger demand during the booking period, re-fleeting adjusts the capacity by re-assigning aircraft types to flight legs to reduce the operational costs and increase the passenger revenues. Sherali et al. (2005) propose a demand-driven refleeting model that dynamically reassigns aircraft based on the improved demand forecasts so as to maximize the total revenue. In order to preserve the crew assignment, the aircraft reassignment is limited within the same aircraft family. To deal with highly uncertain demand well in advance in departures, Sherali and Zhu (2008) propose a two-stage stochastic mixed integer programming approach. In the first stage, they only assign aircraft families to each flight leg. After receiving the improved demand forecasts, refleeting process within each family is conducted for each forecasted demand realization in the second stage. Jarrah et al. (2000) also present a refleeting model while limiting the number of changes to the original fleet assignment. In the re-fleeting approach, they re-assign the fleet types to scheduled flight legs with minor adjustments. They only allow the swapping aircraft within the same aircraft family or put restrictions on the changes to the original schedule, because airlines wish to preserve the crew schedules which have to be constructed one or two months prior to flight departures due to the crew regulations. Similarly, in our proposed study, we wish to leave the aircraft paths unchanged in order to eliminate additional costs of adjustment. We assign aircraft types to these paths.

Rexing et al. (2000) model the basic fleet assignment problem with time windows in order to improve the fleet assignment solutions. Mercier and Soumis (2007) show that allowing changes on the scheduled departure times within an integrated aircraft routing and crew scheduling model yields significant cost savings. Papadakos (2009) also includes re-timing possibilities for aircraft routing and crew scheduling. Sherali et al. (2013) propose a model that integrates the schedule design and fleet assignment while allowing flight departure times vary within time windows. They claim that retiming approach increases the connection opportunities for passengers and generates more profitable schedules. In general, the flexibility in departure times is achieved with multiple discretized copies of the flight legs within specified time intervals. As opposed to discrete time units, in our proposed study, we allow the departure time of each flight continuously vary within a given time interval. In addition to flexible departure times, we control the cruise times to provide more opportunity to satisfy the passenger connections.

When planned schedule is disrupted, aircraft routings, crew pairings and passenger itineraries have to be recovered. Traditional airline recovery approaches ignore passenger itineraries until the end of the recovery process so recovered schedule may not be feasible for passenger flow. Maher (2015) considers the passenger flows in integrated schedule, aircraft and crew recovery problems. Burke et al. (2010) observe the effect of a randomly generated disruption on KLM airlines' schedule. They obtain recovery by simultaneous flight retiming and aircraft rerouting.
Most scheduling models ignore the unexpected flight delays to limit the complexity. However, flight delays may result in disruption on the passenger and aircraft connections, thereby lead to loss of time and customer goodwill. This is the reason why many airlines have been recently interested in generating a robust schedule with improved capability in response to variability in airline operations. Weide et al. (2010) produce solutions that are robust to variability in airline operations for aircraft routing and crew pairing problem by penalizing aircraft changes under short connection times. A common approach in coping with delays is to leave idle times. Ahmadbeygi et al. (2010) and Chiraphadhanakul and Barnhart (2013) propose models minimizing delay propagation in entire network by redistributing the existing slack. They adjust flight departure times to provide more slack over critical connections and draw excess slack from others. Lan et al. (2006) propose a mixed integer programming model, which minimizes the delay propagation by allowing changes in aircraft assignment, and develop an approach to reduce the passenger misconnections by re-timing the flight departure times for a fixed fleet assignment. More recently, Dunbar et al. (2014) incorporate delay scenarios within the aircraft routing and crew pairing problems while re-timing of flight departures. In a similar way, they use re-timing approach to provide slack across the connections so as to minimize delay propagation. To capture the uncertainties in flight block times, Arrkan and Deshpande (2012) model the flight block time distribution and provide a method for estimating the schedule on-time arrival probability. Sohoni et al. (2011) propose models that capture uncertainties related with block time through chance constraints and consider flight re-timing. The aim is to maximize expected profit while improving on-time performance measure and passengers' service level. Duran et al. (2015) design a robust flight schedule incorporating cruise time controllability. They propose a model which captures the variability in flight duration due to the airport congestions via a random variable representing noncruise times.

In the existing literature, cruise time has been often taken as a fixed parameter, although there occur options of flying faster to increase passenger connection possibilities and flying slower for conservation of fuel as discussed in Cook et al. (2009). Bertsimas et al. (2010) decide on an optimum combination of flow management actions, including ground holding, rerouting, speed control and airborne holding. They control the speed through adjustments in the time spent in each en route sector. However, they do not consider fuel burn and carbon emission costs associated with speed adjustment. Sherali et al. (2006) state that airline optimization models are quite sensitive to fuel burn. Tetzloff and Crossley (2010) address environmental and economic considerations by developing a model determining the new and existing aircraft assignment such that all passenger demand is met. The major difficulty of incorporating cruise speed control is that the fuel burn and carbon emission cost functions are nonlinear functions of cruise speed. Consequently, handling the nonlinear model in a reasonable amount of time is critical for solving such problems. To overcome this difficulty, we use mixed integer second order cone programming as discussed in Aktürk et al. (2014).

The contributions of this paper include the following:

- We consider the fuel burn and $\mathrm{CO}_{2}$ emissions costs associated with adjusting cruise speed to ensure the passenger connections. Therefore, we may prefer to assign a fuel efficient but smaller aircraft to an aircraft path involving critical passenger connections in albeit of an additional cost of spilled passengers.

- We assign fleet types by considering not only flight timing and passenger demand, as commonly done in the literature, but also operational expenses, such as fuel burn and carbon emission costs. 
- The proposed model has nonlinear fuel and emission cost terms and chance constraints to ensure the passenger connections with a desired probability. To handle nonlinearity, we utilize mixed integer second order cone programming (MISOCP).

- We devise a two-stage algorithm, which decomposes the problem into two planning stages such as aircraft-path assignment and robust schedule design, and then solves them sequentially.

- We present extensive computational results using a schedule for a major U.S. airline to demonstrate the high quality performance of the two-stage algorithm.

We organize the remainder of this paper as follows. In Section 2, the framework of the problem is briefly described. A proposed mathematical model is given with a numerical example. Section 3 is devoted to the conic reformulation of the proposed model in detail. We describe our approach to simplify the problem and two-stage algorithm in Section 4. We report the computational results in Section 5. Finally, in Section 6, we conclude with remarks and outline possible research directions arising from this study.

\section{Problem definition}

In this study, we would like to assign fleet types to given flight paths during the booking period. We consider not only flight timing and passenger demand, as commonly done in the literature, but also operational expenses, such as fuel burn and carbon emission costs associated with cruise speed adjustment to ensure the passenger connections. Therefore, we propose a bi-criteria optimization model. The first objective is to reduce the airline overall operational cost, where as the second objective is to increase the passenger connection probabilities under the non-cruise time uncertainties due to the airport congestions. We satisfy passenger connections in the entire network through the chance constraints. To achieve desirable connection probabilities, we allow minor adjustments on the flight departure times by redistributing the existing slack over vulnerable connections and removing excess slack from the remaining connections. Simultaneously, we control the cruise speed to ensure desirable connection probabilities. However, there occur additional fuel and $\mathrm{CO}_{2}$ emission costs associated with speed adjustment. In order to reduce the fuel expenses, one approach is to assign a fuel efficient aircraft to an aircraft path with critical passenger connections. On the other hand, fuel efficient but a smaller aircraft may cause an additional cost of spilled passengers or lost revenue due to under-capacity. In this study, our main claim is that we may compensate the cost of spilled passengers by assigning fuel efficient aircraft, when we consider the fuel burn and $\mathrm{CO}_{2}$ emissions costs associated with adjusting cruise speed to ensure the passenger connections.

In the booking period, airlines wish to keep generated schedule close to the original one designed well in advance. Because, even minor changes on flight schedule or aircraft routes may lead to a massive disruption on the aircraft, passenger and crew connections. Therefore, we work with all aircraft paths which have been determined through the airlines' scheduling choices. We define an aircraft path to be a sequence of flights operated by an individual aircraft in a given time period. All input paths form a complete partition of all the flights.

The notation used throughout the paper is given below: Parameters

$\begin{array}{ll}T & \text { set of aircraft types } \\ P & \text { set of paths } \\ J & \text { set of flights } \\ J_{p} & \text { set of flights in path } p \in P \\ N^{t} & \text { available number of aircraft of type } t \in T \\ C A P^{t} & \text { number of seats in aircraft of type } t \in T\end{array}$

$\left[f_{i}^{t, l}, f_{i}^{t, u}\right]$ time window for cruise time of flight $i \in J$ with aircraft $t \in T$

$A_{i} \quad$ random variable representing the non cruise time of flight $i \in J$

$E\left[A_{i}\right] \quad$ expected non-cruise time of flight $i \in J$

PAIR set of pairs of consecutive flights of the same aircraft

$T A_{i j}^{t} \quad$ turnaround time needed to prepare aircraft $t \in T$ between flights $i, j \in$ PAIR

$D_{i} \quad$ number of passenger demand of each flight $i \in J$

$\mathrm{Csp}_{i} \quad$ cost of spilled passengers of flight $i \in J$

$c_{\text {fuel }} \quad$ cost of fuel per $\mathrm{kg}$ of aircraft fuel consumption

$\mathrm{C}_{\mathrm{CO}_{2}} \quad$ cost of emission per $\mathrm{kg}$ of aircraft $\mathrm{CO}_{2}$ emission

$I^{t} \quad$ unit idle time cost of aircraft of type $t \in T$ in dollars per minute

$P_{i} \quad$ set of flights that have a passenger connection with flight $i \in J$

$T P_{i j} \quad$ turntime needed to connect passengers between flights $i \in J, j \in P_{i}$

minimum service level for each passenger connection between flights $i \in J$ and $j \in P_{i}$

$\gamma_{i j}^{d}$

$w_{i j}$

$J, j \in P_{i}$

$\left[v_{i}^{l}, v_{i}^{u}\right] \quad$ time window for the departure time of flight $i \in J$

$\mathrm{O}_{i} \quad$ origin of flight $i \in J$

$D n_{i} \quad$ destination of flight $i \in J$

$B \quad$ set of airports

$e_{b} \quad$ airport congestion coefficient for airport $b \in B$

Decision Variables

$z_{p}^{t} \quad 1$ if aircraft of type $t \in T$ is assigned to path $p \in P$, and 0 , otherwise.

$x_{i} \quad$ departure time of flight $i \in J$

$f_{i}^{t} \quad$ cruise time of flight $i \in J$ with aircraft type $t \in T$

$S_{i}^{t} \quad$ idle time of flight $i \in J$ with aircraft type $t \in T$

$\gamma_{i j} \quad$ service level for passenger connections between flights $i$ $\in J$ and $j \in P_{i}$, i.e., probability that passengers from flight $i$ can connect to any follow-on flight $j \in P_{i}$

\subsection{Mathematical model} low.

The proposed nonlinear mathematical model is provided be-

$\min F 1: \sum_{p \in P} \sum_{i \in J_{p}} \sum_{t \in T} C_{\text {fuel\& } C_{2}}^{i, t}\left(f_{i}^{t}\right)$

$+\sum_{p \in P} \sum_{i \in J_{p}} \sum_{t \in T} C s p_{i} \cdot \max \left(D_{i}-C A P^{t}, 0\right) \cdot z_{p}^{t}$

$+\sum_{i \in J} \sum_{t \in T} S_{i}^{t} \cdot I^{t}$

$\max \quad F 2: \sum_{i \in J} \sum_{j \in P_{i}} w_{i j} \cdot \gamma_{i j}$

subject to

$\sum_{t \in T} z_{p}^{t}=1 \quad p \in P$

$\sum_{p \in P} z_{p}^{t} \leq N^{t} \quad t \in T$

$\operatorname{Pr}\left[A_{i}+\sum_{t \in T} f_{i}^{t} \leq x_{j}-x_{i}-T P_{i j}\right] \geq \gamma_{i j} \quad i \in J, j \in P_{i}$ 
$\gamma_{i j} \geq \gamma_{i j}^{d} \quad i \in J, j \in P_{i}$

$f_{i}^{t, l} \cdot z_{p}^{t} \leq f_{i}^{t} \leq f_{i}^{t, u} \cdot z_{p}^{t} \quad p \in P, i \in J_{p}, t \in T$

$x_{j}-x_{i}-\sum_{t \in T} T A_{i j}^{t} \cdot z_{p}^{t}-\sum_{t \in T} f_{i}^{t}-E\left[A_{i}\right]-\sum_{t \in T} S_{i}^{t}=0$

$$
(i, j) \in \text { PAIR }
$$

$v_{i}^{l} \leq x_{i} \leq v_{i}^{u} \quad i \in J$

$w_{i}^{l} \leq x_{i}+\sum_{t \in T} f_{i}^{t}+E\left[A_{i}\right] \leq w_{i}^{u} \quad i \in J$

$S_{i}^{t} \leq M \cdot z_{p}^{t} \quad p \in P, i \in J_{p}, t \in T$

$S_{i}^{t} \geq 0 \quad i \in J, t \in T$

$z_{p}^{t} \in\{0,1\} \quad p \in P, t \in T$

The most common objective that airlines minimize is the overall operational cost. However, a schedule that minimizes the operational cost may not ensure a high service level for passengers' connections. Thus, in this study, we consider a bi-criteria problem. The first objective minimizes the overall operational cost and the second objective maximizes the overall service level for passenger connections through the entire network. The sum of fuel cost, $\mathrm{CO}_{2}$ emission cost, spilled passengers cost and idle time cost over all flights in the network constitute airline's operational cost. Moreover, overall service level for passenger connections is the weighted average of the service level for each passenger connection in the network. Constraint (3) guarantees that each path is assigned to exactly one aircraft type. Constraint (4) limits the number of employed aircraft by $N^{t}$. Constraint (5) is a chance constraint to ensure each passenger connection with a desirable service level. We require the probability that passengers from flight $i$ can connect to any follow-on flight $j \in P_{i}$, to be greater than or equal to service level variable $\gamma_{i j}$. Constraint (6) applies a desired lower bound on the service level variable $\gamma_{i j}$. Constraint (7) applies cruise time upper and lower bound for each flight. Constraint (8) guarantees minimum aircraft turnaround time between two consecutive flights. In (9) and (10), we want the departure and arrival times of each flight to be within the time intervals which have already been determined by the airline. Time window constraints (9) and (10) can be used to restrict the departure and arrival times of flights within the airlines's own slot times. In this manner, any penalty cost of flights arriving or departing outside of the allocated slot times can be eliminated. Constraint (11) is a Big-M constraint to eliminate the possible nonlinearity. Constraints (12) and (13) define the domain of the variables.

To solve this bi-criteria optimization problem, we use the $\epsilon$ constraint approach (T'kindt and Billaut, 2006). In this approach, we will solve the problem of minimizing $F 1$ for a given lower bound on $F 2$. We add the following service level bound constraint into the proposed model.

$\sum_{i \in J} \sum_{j \in P_{i}} w_{i j} \cdot \gamma_{i j} \geq \gamma$

In (14), we want the overall service level be greater than or equal to the desired level, $\gamma$. The $\epsilon$-constraint method has been widely used in the literature, because the decision maker can interactively specify and modify the service level bounds and analyze the influence of these changes on the total operational cost.

\subsubsection{Service level}

In this paper, we generate a schedule which is robust to variability at congested airports by increasing passenger service levels. Random variable representing non-cruise times arises in chance constraints (5). Constraint (5) ensures the minimum passenger connection time $T P_{i j}$ between the arrival of flight $i$ and departure of flight $j$ with a probability greater than or equal to $\gamma_{i j}$. In constraint (6), we wish to keep the service level $\left(\gamma_{i j}\right)$ for each connection greater than or equal to desired lower bound $\gamma_{i j}^{d}$, e.g., an airline may prefer to satisfy the international connections with higher probabilities.

One of our aims is to maximize the overall service level through the entire network. The overall service level is calculated as the weighted average of the service level of each connection as defined in the second objective function of $F 2$ in (2). To increase the service level for each passenger connection, we allow changes on the flight departure times within the time window given in Constraint (9). In addition, in response to high variability at the congested airports, we can set a higher cruise speed to ensure the passenger connections with a higher service level. Consequently, we wish to achieve a robust schedule, which is less susceptible to unexpected flight delays due to airport congestions.

\subsubsection{Distribution of non-cruise times}

Deterministic approaches model the random parameters by their expected values. However, expected values may be too far from the certain realizations in practice, thereby resulting plan may perform poorly. One of the uncertainties in flight appears in non-cruise stage, because actual non-cruise time may take longer than expected due to the airport congestions. We represent the non-cruise time of each flight by a random variable.

Arıkan and Deshpande (2012) show that the log-Laplace distribution provides a good-fit to the block time of a flight. For each flight $i \in J$, we assume random variable $A_{i}$ representing the noncruise time has $\log$-Laplace distribution with a scale parameter, $e^{\alpha}$ and the tail parameter, $1 / \beta_{i}$. For each flight $i \in J$, we define $\beta_{i}$ as a function of the congestion coefficients of the origin and destination airports of flight $i$. We express $\beta_{i}$ as

$\beta_{i}=\beta \cdot\left(e_{O_{i}}\right)^{2} \cdot\left(e_{D n_{i}}\right)^{2}$

where $O_{i}$ and $D n_{i}$ are the origin and destination airports of flight $i$ $\in J$, respectively. Variability is higher at congested airports. Moreover, the mean of the non-cruise times increases at the congested airports. Duran et al. (2015) provide the mean of the random variable $A_{i}$ as follows

$$
E\left[A_{i}\right]=\frac{e^{\alpha}}{\left(1-\beta_{i}\right) \cdot\left(1+\beta_{i}\right)} .
$$

\subsubsection{Fuel and $\mathrm{CO}_{2}$ emission cost functions}

In this study, we utilize the idea of aircraft speed control to ensure passenger connections. However, speed decisions affect fuel burn. To estimate the fuel burn, we use the cruise stage fuel flow model developed by the Base of Aircraft Data (BADA) (EUROCONTROL, 2012) which is discussed in detail in Appendix. Fuel burn $(\mathrm{kg})$ as a function of cruise time $f_{i}^{t}(\mathrm{~min})$ can be calculated as

$F_{i}^{t}\left(f_{i}^{t}\right)=c_{1}^{i, t} \cdot \frac{1}{f_{i}^{t}}+c_{2}^{i, t} \cdot \frac{1}{\left(f_{i}^{t}\right)^{2}}+c_{3}^{i, t} \cdot\left(f_{i}^{t}\right)^{3}+c_{4}^{i, t} \cdot\left(f_{i}^{t}\right)^{2}$

where coefficients $c_{j}^{i, t}>0, j=1, \ldots, 4$, are expressed in terms of aircraft specific fuel consumption and drag as well as the mass of 
aircraft, air density and gravitational acceleration. Note that, $F_{i}^{t}\left(f_{i}^{t}\right)$ is a convex function of $f_{i}^{t}>0$.

Fuel cost for flight $i$ operated by aircraft type $t$ can be calculated as

FuelCost $_{i}^{t}\left(f_{i}^{t}\right)=c_{\text {fuel }} \cdot\left(F_{i}^{t}\left(f_{i}^{t}\right)\right)$

where $c_{\text {fuel }}$ is the unit price for jet fuel $(\$ / \mathrm{kg})$.

International Civil Aviation Organization (ICAO) developed standards for aircraft engine emissions, which forces airlines to put more emphasis on calculation of emissions. EUROCONTROL (2001) states that $\mathrm{CO}_{2}$ emissions are approximately 3.15 times the weight of fuel consumed. Therefore, cost of $\mathrm{CO}_{2}$ emission can be expressed as a function of cruise time as

EmissionCost $_{i}^{t}\left(f_{i}^{t}\right)=c_{\mathrm{CO}_{2}} \cdot k \cdot F_{i}^{t}\left(f_{i}^{t}\right)$

where $c_{\mathrm{CO}_{2}}$ is the unit cost of $\mathrm{CO}_{2}$ emission $(\$ / \mathrm{kg})$ and $k$ is $\mathrm{CO}_{2}$ emission constant.

For each $p \in P, i \in J_{p}, t \in T$, we combine the fuel and emission cost functions and redefine them as

$C_{\text {fuel\& } \mathrm{CO}_{2}}^{i, t}\left(f_{i}^{t}\right)= \begin{cases}\left(c_{\text {fuel }}+c_{\mathrm{CO}_{2}} \cdot k\right) & \\ \cdot\left(c_{1}^{i, t} \cdot \frac{1}{f_{i}^{t}}+c_{2}^{i, t} \cdot \frac{1}{\left(f_{i}^{t}\right)^{2}}+c_{3}^{i, t} \cdot\left(f_{i}^{t}\right)^{3}\right. & \\ \left.+c_{4}^{i, t} \cdot\left(f_{i}^{t}\right)^{2}\right) & \text { if } z_{p}^{t}=1 \\ 0 & \text { if } z_{p}^{t}=0\end{cases}$

so that if an aircraft of type $t$ is not assigned to path $p$, then $C_{\text {fuel } \& \mathrm{CO}_{2}}^{i, t}\left(f_{i}^{t}\right)=0 i \in J_{p}$.

Fuel consumption function $F_{i}^{t}\left(f_{i}^{t}\right)$ is minimized at Maximum Range Cruise (MRC) speed. Although the most fuel efficient case is to fly at MRC speed, airlines sometimes prefer to fly at a higher speed to ensure the aircraft and passengers' connections.

\subsubsection{Objectives}

In this paper, we deal with a bi-criteria optimization problem of simultaneously minimizing airline's operational costs, denoted as $F 1$ in (1), and maximizing service level for passenger connections, denoted as F2 in (2), in the proposed nonlinear mathematical model. To satisfy the passenger connections with a higher service level, we may employ both idle time insertion and cruise time compression, or only use one of them based on their impact on operational cost. In order to decrease fuel and emission costs, we may assign a fuel efficient but smaller aircraft to flights whose cruise times need to be compressed. Although we spill some of the passengers, we may compensate the cost of spilled passengers by conservation of fuel. Therefore, we simultaneously consider these interrelated cost terms such as fuel and emission costs, idle time insertion costs and cost of spilled passengers to achieve a minimum cost schedule.

For flight $i$, aircraft type $t$, we express idle time cost function as

$C_{i d l e}^{i, t}\left(S_{i}^{t}\right)=S_{i}^{t} \cdot I^{t}$

Similarly, we represent cost of spilled passengers with a linear function of the number of passengers who cannot be accommodated and turned away by the airlines due to the insufficient seat capacity of aircraft. For flight $i$, aircraft type $t$, spilled passenger cost can be expressed as

$C_{\text {spilled }}^{i, t}=C s p_{i} \cdot \max \left(D_{i}-C A P^{t}, 0\right) \cdot z_{p}^{t}$

\subsection{Numerical example}

In this section, a numerical example is provided to illustrate how fuel consumption and $\mathrm{CO}_{2}$ emission of aircraft affect the assignment decisions and how the cruise time controllability and idle time insertion can be utilized to generate a robust schedule.
In this small example, we consider two given paths operated by two different aircraft. Table 1 shows the tail numbers and flight numbers along with the origin and destination airports, planned departure times, planned block and arrival times, actual arrival times, turnaround times, and demand of flights. Two flights with the same flight number, 336, represents a through flight that includes one or more intermediate airports between the origin and destination airports. The first path including flights 2303, 2336, 1053 and 336 is operated by Boeing 737500 and the second path is flown by MD 83. While aircraft is flying at MRC speed, fuel burn rates of B737 500 and MD 83 are estimated as $29 \mathrm{~kg} / \mathrm{min}$ and $40 \mathrm{~kg} / \mathrm{min}$, respectively. The fuel burn rate is calculated using the fuel flow model of BADA as in Eq. (46) in Appendix.

In daily operations, some flights may not be operated as planned. One reason is that a flight may take longer than the expected duration. In the published schedule, let's assume that $25 \mathrm{~min}$ of the flight duration is planned non-cruise time and remaining is planned cruise time. However, non-cruise time has an expected value of 28 min for flight 2303, when we represent noncruise times with a log-Laplace random variable. We calculate the expected value as in Eq. (15) with $\alpha$ taken as $\log (20)$ and $\beta$ taken as 0.05 . Therefore, actual flight duration is $2 \mathrm{~h} 33 \mathrm{~min}$, so that the actual arrival time is 9:08 which is 3 min later than planned arrival time. In the published schedule given in Table 1 , some time is left between the arrival and departure times of consecutive flights of each aircraft. If this time is not enough to prepare an aircraft for the next flight, there will be some delays on departure of next flight. Such delays may result in passenger misconnections. On the other hand, if this time is longer, there exists idle times between the consecutive flights. For example, time between planned arrival of flight 1131 and planned departure of flight 1339 is $65 \mathrm{~min}$. An aircraft requires $36 \mathrm{~min}$ to be prepared for next flight 1339 . Therefore, $29 \mathrm{~min}$ between these consecutive flights are enough to capture 3 min of delay on arrival of flight 1131. In other words, $26 \mathrm{~min}$ of idle time remains between flights 1131 and 1339 .

The time-space network diagram of the published schedule in Table 1 is given in Fig. 1. In Fig. 1 continuous arcs represent planned flights, where the dashed arcs represent actual flight times. The blue and red arcs in Fig. 1 are for aircraft N531AA and N454AA, respectively. Turnaround times of the aircraft are represented by the continuous ground lines and idle times are represented by the dashed ground lines.

Fig. 1, departure of the first leg of flight 336 is delayed, since there is not enough time between actual arrival of flight 1053 and planned departure of flight 336 to prepare an aircraft for flight 336. On the other hand, there exist 18 min idle times before departure of flight 1053. Therefore, published schedule needs a better utilization of idle times by re-timing departure times as already discussed in Ahmadbeygi et al. (2010). Re-timing approach can be used to increase passenger connection possibilities. We also use the idea of speeding up some aircraft especially at congested airports to ensure passenger connections at a desired service level. However, we should consider adverse effect of speeding up aircraft on fuel and carbon emission costs. If we assign a fuel efficient aircraft to a flight, reduction in fuel cost may compensate the cost of idle time insertion. On the other hand, this assignment may incur an additional cost of spilled passengers. The objective function of the integrated model (IM) considers these four conflicting cost components of the schedule. The new schedule achieved by IM is provided in Fig. 2.

In Fig. 2, we see that aircraft assignments among two paths are switched compared to the published schedule. Red and blue arcs in Fig. 2 are for aircraft N531AA and N454AA, respectively. integrated model assigns fuel efficient aircraft B737 500 to the second path. Our approach compresses cruise time durations of flights 2441, 1986, 1872 and 1131 by 10,10, 12 and $12 \mathrm{~min}$, respectively. 
Table 1

Published schedule.

\begin{tabular}{llllllllll}
\hline Tail \# & Flight \# & From & To & Plan. dep. & Plan. dur. & Plan. arr. & Act. arr. & TA & Demand \\
\hline N531AA & 2303 & ORD & DFW & $6: 35$ & $2: 30$ & $9: 05$ & $9: 08$ & $0: 48$ & 121 \\
& 2336 & DFW & ORD & $10: 00$ & $2: 35$ & $12: 35$ & $12: 38$ & $0: 49$ & 117 \\
& 1053 & ORD & ATL & $13: 45$ & $2: 05$ & $15: 50$ & $15: 50$ & $0: 47$ & 110 \\
& 336 & ATL & ORD & $16: 30$ & $2: 10$ & $18: 40$ & $18: 47$ & $0: 25$ & 120 \\
& 336 & ORD & LGA & $19: 20$ & $2: 15$ & $21: 35$ & $21: 37$ & & 112 \\
N454AA & 2441 & ORD & ATL & $6: 45$ & $2: 10$ & $8: 55$ & $8: 55$ & $0: 33$ & 118 \\
& 1986 & ATL & ORD & $09: 40$ & $2: 15$ & $11: 55$ & $11: 55$ & $0: 36$ & 121 \\
& 1872 & ORD & DFW & $12: 35$ & $2: 30$ & $15: 05$ & $15: 08$ & $0: 34$ & 129 \\
& 1131 & DFW & ORD & $15: 50$ & $2: 35$ & $18: 25$ & $18: 28$ & $0: 36$ & 122 \\
\hline
\end{tabular}

LGA

DFW
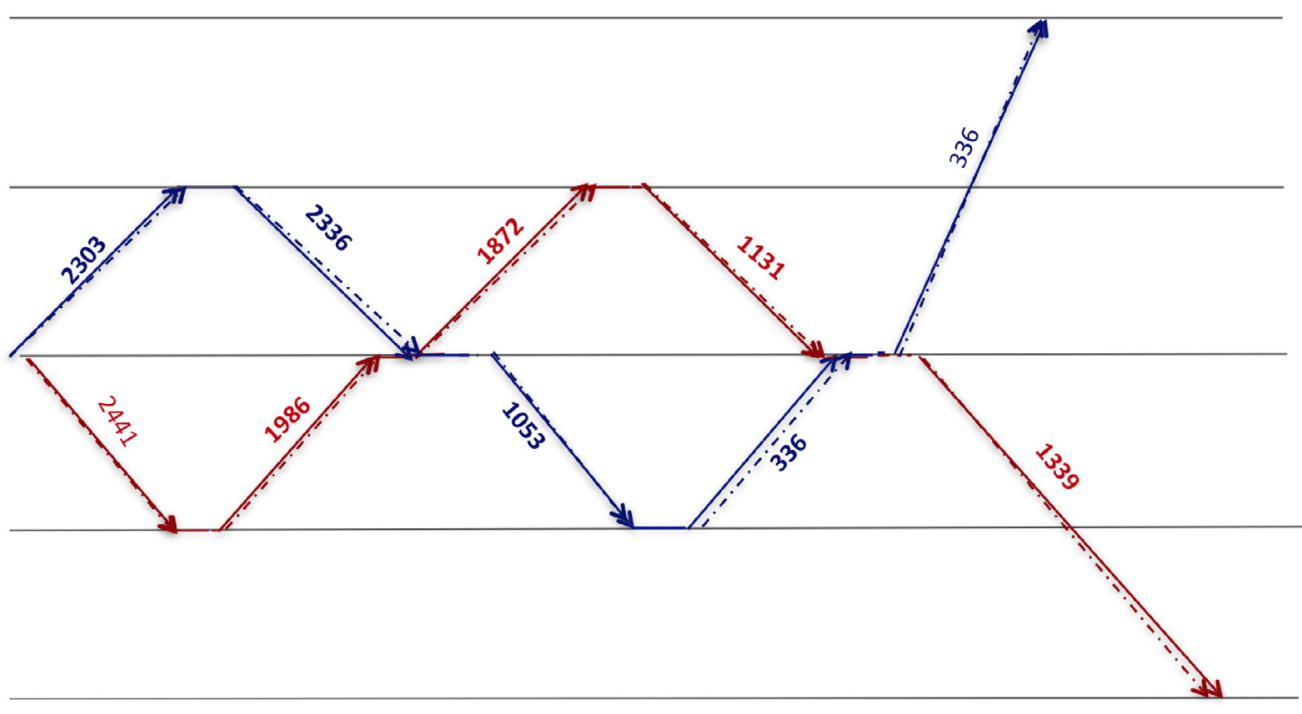

SAN

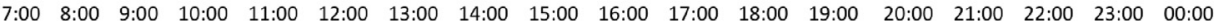

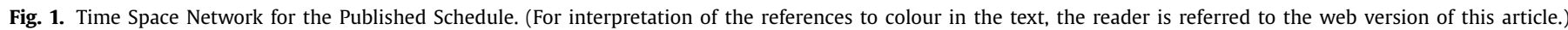

The reason of speeding up the aircraft is that, passengers of flight 1131 have connections to the second leg of flight 336. The crucial point is that total compression amount of $44 \mathrm{~min}$ is not imposed upon only on flight 1131 . Due to the nonlinearity of fuel cost function, it is more beneficial to allocate the required compression to multiple flights. In Fig. 2, continuous arcs represent flights operated under the MRC speed, where dashed lines refer to flights with compressed cruise times.

Speeding up aircraft may not be enough to satisfy passenger connections at a desired service level. Therefore, $16 \mathrm{~min}$ of idle time is also inserted before flight 336 to satisfy passenger connections between flights 1131 and 336. The reason for utilizing both speed control and idle time insertion is that speeding up the aircraft might be cheaper than idle time insertion up to a point, and then idle time insertion might be cheaper due to the nonlinearity of fuel cost function.

We compare the performance of new schedule with the published schedule in terms of operational costs. Fuel and $\mathrm{CO}_{2}$ emission costs are calculated as explained in Eqs. (17) and (18), respectively. Idle time costs are calculated by multiplying total idle time with the unit idle cost of aircraft, which is given in Table 5. The cost of spilled passengers are also calculated as multiplying total number of spilled passengers with the unit cost which is calculated as in Eq. (44).

Table 2 shows the improvements in fuel and emission cost, idle time insertion cost and total cost compared to the published schedule. We assume that aircraft speed is constant in the published schedule. In our approach, we use the option of flying faster
Table 2

Cost comparison.

\begin{tabular}{rllll}
\hline & $\begin{array}{l}\text { Fuel \& emission } \\
\text { cost }(\$)\end{array}$ & $\begin{array}{l}\text { Idle } \\
\text { cost }(\$)\end{array}$ & $\begin{array}{l}\text { Spilled } \\
\text { cost }(\$)\end{array}$ & $\begin{array}{l}\text { Total } \\
\text { cost }(\$)\end{array}$ \\
\hline Published schedule & 56,196 & 11,173 & 0 & 67,369 \\
Integrated model (IM) & 54,841 & 4,766 & 708 & 60,315 \\
\hline
\end{tabular}

to satisfy the passenger connections. However, speeding up the aircraft incurs additional fuel and emission costs. By considering fuel burn and carbon emission costs, integrated model switches the assignment of aircraft types among two paths compared to published schedule. By this fleet assignment, we obtain $2.5 \%$ cost saving in fuel consumption and $\mathrm{CO}_{2}$ emissions compared to the published schedule. Total fuel and $\mathrm{CO}_{2}$ emission costs for the published schedule and new schedule are $\$ 56,196$ and $\$ 54,841$, respectively. On the other hand, this assignment spills 31 passengers of total 1,216 passengers, which costs $\$ 708$. In addition to speeding up aircraft, to satisfy the passenger connections at $90 \%$ service level, $17 \mathrm{~min}$ and $16 \mathrm{~min}$ of idle times are also inserted before flight 1053 and second leg of flight 336. Therefore, there exists total $33 \mathrm{~min}$ of idle time in the new schedule, whereas there exists $80 \mathrm{~min}$ of idle time in the published schedule. Our proposed model eliminates the unnecessary idle times and reallocates the required amount of slack among the flights with controllable cruise time decisions so that passenger connections are satisfied at desired service level. It follows that new schedule results in $57 \%$ improvement in idle time cost, where the costs of the idle time in the published sched- 


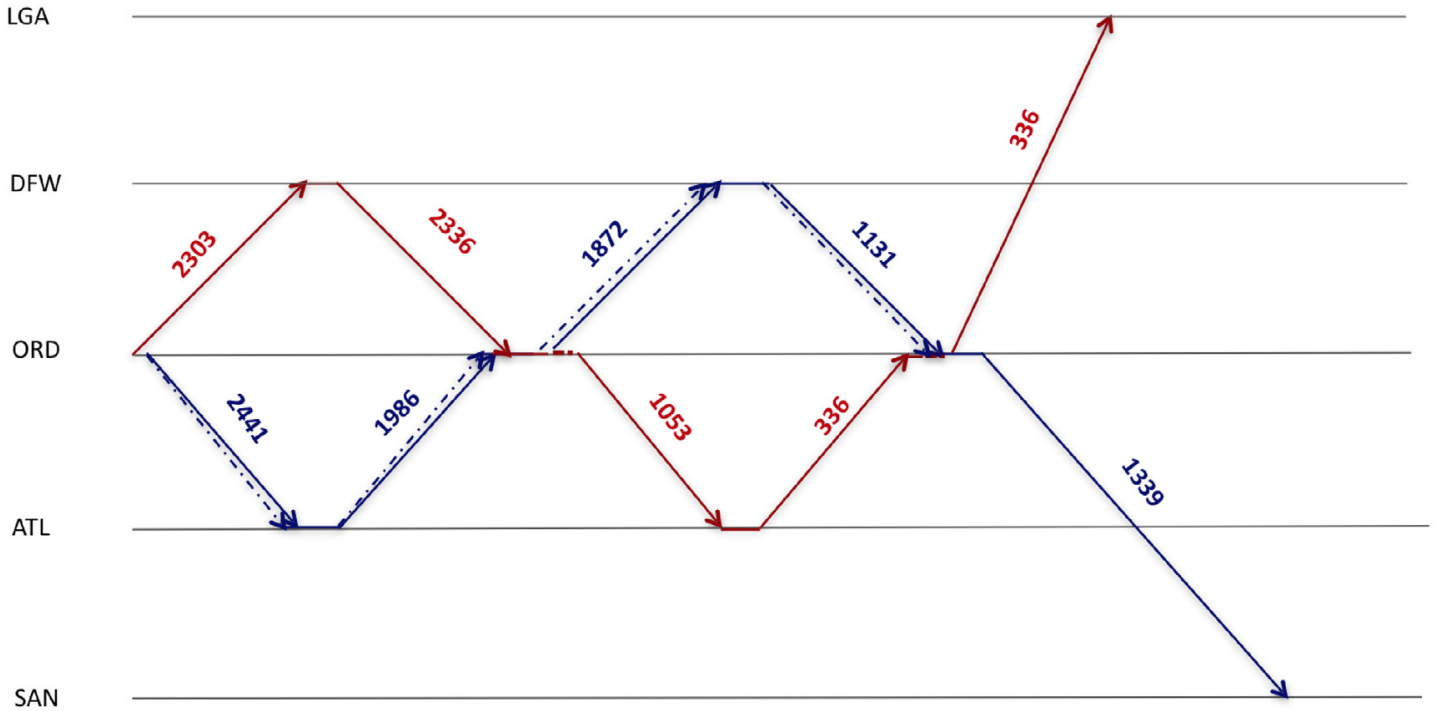

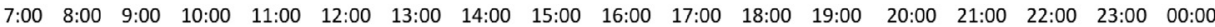

Fig. 2. Time Space Network - by IM.

ule and new schedule are $\$ 11,173$ and $\$ 4,766$, respectively. In total, operational cost saving is around $10 \%$ compared to the published schedule.

\section{Conic reformulation of integrated model}

Our model involves nonlinear fuel and carbon emission cost terms in the objective function and chance constraints. Nonlinear mixed integer optimization often requires significant computation time to achieve optimal or suboptimal solutions. To reduce the computation time, in this section, we utilize conic quadratic programming.

\subsection{Conic representation of chance constraints}

In this study, we ensure passenger connections through chance constraints with a desired service level. In the literature, most of the studies handle the chance constraints with approximation methods. In this section, we show how to reformulate the chance constraints via second order conic inequalities.

The random variable representing non-cruise time of a flight arises in chance constraints. For each flight $i \in J$, we assume that random variable $A_{i}$ representing non-cruise time has Log-Laplace distribution. The density and quantile function of the Log-Laplace distribution can be found in Appendix.

Constraint (5) can be expressed using the quantile function of the probability distribution of random variable $A_{i}$ with parameters $e^{\alpha}$ and $1 / \beta_{i}$ as follows:

$\frac{e^{\alpha}}{2^{\beta_{i}} \cdot\left(1-\gamma_{i j}\right)^{\beta_{i}}} \leq x_{j}-x_{i}-T P_{i j}-\sum_{t \in T} f_{i}^{t}, \quad$ if $\quad \frac{1}{2} \leq \gamma_{i j} \leq 1$

In this study, we wish to keep each service level greater than or equal to $50 \%$, this is the reason why we consider the quantile function for $0.5 \leq \gamma_{i j}$ in constraint (21). Duran et al. (2015) achieve the convexity of the expression on the left hand side of inequality (21). Then, Proposition 1 gives the conic representation of the constraint (21).
Proposition 1. For $i \in J, j \in P_{i}$, if $0<\beta_{i}<1$ and $\frac{1}{2} \leq \gamma_{i j} \leq 1$, then constraint (21):

$\frac{e^{\alpha}}{2^{\beta_{i}} \cdot\left(1-\gamma_{i j}\right)^{\beta_{i}}} \leq x_{j}-x_{i}-T P_{i j}-\sum_{t \in T} f_{i}^{t}$,

can be represented via second order conic inequalities.

Proof. The proof is similar to the proof of Proposition 2 in Duran et al. (2015). In their study, fleet types are taken as fixed parameters and cruise times are represented by $f_{i}$ for each flight $i \in J$. In this study, cruise times represented by $f_{i}^{t}$ vary among fleet types. Therefore, in their proof, we can replace $f_{i}$ with $\sum_{t \in T} f_{i}^{t}$, since $f_{i}^{t}=0$ if the $z_{p}^{t}=0$. Then, we obtain the following hypograph.

Let the constant $\lambda=\frac{e^{\alpha}}{2^{\beta_{i}}}$ and $\beta_{i}=\frac{a_{i}}{b_{i}}$ for integers $a_{i}$ and $b_{i}$. Choose $l$ such that $l=\left\lceil\log _{2}\left(a_{i}+b_{i}\right)\right\rceil$. Then, chance constraints in the original formulation can be replaced by the following constraints for $i \in J, j \in P_{i}$ :

$$
\begin{aligned}
\left(x_{j}-x_{i}-T P_{i j}-\sum_{t \in T} f_{i}^{t}\right) & =\sigma_{i j} \\
\bar{\gamma}_{i j} & =1-\gamma_{i j} \\
\sigma_{i j}{ }^{b_{i}} \cdot \bar{\gamma}_{i j}{ }^{a_{i}} & \geq\left(\sqrt[2^{l}]{\lambda^{b_{i}}}\right)^{2^{l}}
\end{aligned}
$$

Due to Ben-Tal and Nemirovski (2001), the hypograph of the geometric mean of $2^{l}$ variables can be represented via second order conic inequalities. In (22), it can be seen that $b_{i}$ of the variables equal to $\sigma_{i j}, a_{i}$ of the variables equal to $\bar{\gamma}_{i j}$ and the remaining $2^{l}-a_{i}-b_{i}$ variables can be set to 1 . Hence, it is clearly observed that constraint (22) represents the hypograph of the geometric mean of $2^{l}$ variables. According to Ben-Tal and Nemirovski (2001), the hypograph can be equivalently represented by hyperbolic inequalities of the form,

$u^{2} \leq v_{1} v_{2}, \quad u, v_{1}, v_{2} \geq 0$

which can be represented by the second order conic inequality below

$\left\|\left(2 u, v_{1}-v_{2}\right)\right\| \leq v_{1}+v_{2}$

that concludes the proof. 
3.2. Conic representation of fuel and $\mathrm{CO}_{2}$ cost functions

In this section, we show conic quadratic reformulation of fuel burn and carbon emission cost functions. To simplify the presentation, we drop the indices of the variables and parameters as follows:

$C_{\text {fuel\& } \mathrm{CO}_{2}}(f)=\left\{\begin{array}{cc}\left(c_{f u e l}+c_{\mathrm{CO}_{2}} \cdot k\right)\left(c_{1} \cdot \frac{1}{f}\right. & \\ \left.\quad+c_{2} \cdot \frac{1}{f^{2}}+c_{3} \cdot f^{3}+c_{4} \cdot f^{2}\right) & \text { if } z=1 \\ 0 & \text { if } z=0\end{array}\right.$

$C_{f u e l \& \mathrm{CO}_{2}}(f)$ is discontinuous and therefore its epigraph $E_{F}=$ $\left\{(f, t) \in R^{2}: C_{{\text {fuel } \& \mathrm{CO}_{2}}}(f) \leq t\right\}$ is nonconvex. In the next proposition, we describe how the convexity of $E_{F}$ is obtained. A more detailed information can be found in Aktürk et al. (2014) and Günlük and Linderoth (2010).

Proposition 2. The convex hull of $E_{F}$ can be expressed as

$t \geq\left(c_{\text {fuel }}+k \cdot c_{\mathrm{CO}_{2}}\right)\left(c_{1} \cdot q+c_{2} \cdot \delta+c_{3} \cdot \varphi+c_{4} \cdot \vartheta\right)$

$z^{2} \leq q \cdot f$

$z^{4} \leq f^{2} \cdot \delta \cdot z$

$f^{4} \leq z^{2} \cdot \varphi \cdot f$

$f^{2} \leq \vartheta \cdot z$

in the constraint set. Moreover, each inequalities (24)-(27) can be represented by conic quadratic inequalities.

Proof. Perspective of a convex function $k(f)$ is $z \cdot k(f / z)$ (HiriartUrruty and Lemarećhal, 2001). Since each of the nonlinear terms $\frac{1}{f}, \frac{1}{f^{2}}, f^{3}$ and $f^{2}$ is a convex function for $f \geq 0$, then epigragh of the perspective of each term can be stated as, $\frac{z^{2}}{f} \leq q, \frac{z^{4}}{f^{2}} \leq \delta, \frac{f^{3}}{z^{2}} \leq \varphi$, $\frac{f^{2}}{z} \leq \vartheta$ respectively. Since $z, f \geq 0$, they can be written as stated in the proposition.

Finally, observe that (24) and (27) are hyperbolic inequalities, (25) can be restated as two hyperbolic inequalities

$z^{2} \leq w \cdot f \quad$ and $\quad w^{2} \leq \delta \cdot z$

and (26) can be restated as

$f^{2} \leq w \cdot z \quad$ and $\quad w^{2} \leq \varphi \cdot f$

which can be written as a conic quadratic inequality as described in Section 3.1.

\subsection{Conic reformulation of integrated model}

The model can be reformulated using the hyperbolic inequalities which can be written as conic quadratic inequalities as follows:

$$
\begin{aligned}
& \min \sum_{i \in J} \sum_{t \in T}\left(c_{\text {fuel }}+c_{\mathrm{CO}_{2}} \cdot k\right)\left(c_{1} \cdot q_{i}^{t}+c_{2} \cdot \delta_{i}^{t}+c_{3} \cdot \varphi_{i}^{t}+c_{4} \cdot \vartheta_{i}^{t}\right) \\
& +\sum_{p \in P} \sum_{i \in J_{p}} \sum_{t \in T} \operatorname{Csp} p_{i} \cdot \max \left(D_{i}-C A P^{t}, 0\right) \cdot z_{p}^{t}+\sum_{i \in J} \sum_{t \in T} S_{i}^{t} \cdot I_{i}^{t} \\
& \text { s.to }
\end{aligned}
$$

$$
\text { (3) }-(4)
$$

$\left(z_{p}^{t}\right)^{2} \leq q_{i}^{t} \cdot f_{i}^{t} \quad i \in J_{p}, p \in P, t \in T$

$$
\begin{aligned}
& \left(z_{p}^{t}\right)^{4} \leq\left(f_{i}^{t}\right)^{2} \cdot \delta_{i}^{t} \cdot z \quad i \in J_{p}, p \in P, t \in T \\
& \left(f_{i}^{t}\right)^{4} \leq\left(z_{p}^{t}\right)^{2} \cdot \varphi_{i}^{t} \cdot f_{i}^{t} \quad i \in J_{p}, p \in P, t \in T \\
& \left(f_{i}^{t}\right)^{2} \leq \vartheta_{i}^{t} \cdot z_{p}^{t} \quad i \in J_{p}, p \in P, t \in T \\
& \sigma_{i j}^{b_{i}} \cdot \bar{\gamma}_{i j}^{a_{i}} \geq\left(\sqrt[2^{l}]{\lambda b_{i}}\right)^{2^{l}} \quad i \in J, j \in P_{i} \\
& x_{j}-x_{i}-T P_{i j}-\sum_{t \in T} f_{i}^{t}=\sigma_{i j} \quad i \in J, j \in P_{i} \\
& \bar{\gamma}_{i j}=1-\gamma_{i j} \quad i \in J, j \in P_{i} \\
& \sum_{i \in J} \sum_{j \in P_{i}} w_{i j} \cdot \gamma_{i j} \geq \gamma
\end{aligned}
$$$$
\text { (6) }-(13)
$$

Objective function (28) is slightly different than the original objective function of the proposed model. The original objective, $F 1$ is represented by the new objective and conic constraints (29)-(32). $\epsilon$-constraint approach is implemented by Constraint (36) which imposes a lower bound on $F 2$. The probabilistic constraints (5), are represented by the conic constraints (33)-(35). The remaining constraints are same as the original constraints of the proposed model.

\section{Algorithm for aircraft-path assignment and robust airline scheduling}

Integrated aircraft-path assignment and schedule design is a very complex problem with nonlinear cost terms, chance constraints and binary aircraft assignment decisions. To mitigate the computational difficulties, we utilize mixed integer second order cone programming. However, in real size problems, the vast number of re-timing and re-fleeting decisions may still require problems to be broken into smaller subproblems to efficiently manage tractability. Traditional approaches adopt a sequential planning process. First, decisions of a flight schedule are made, and then assignment of airline's fleet to scheduled flights is determined. Even though sequential planning approach greatly simplifies the solution process, it could create incompatibilities between various stages. For example, a flight schedule in the first stage may not be feasible for aircraft and passenger connections in the subsequent stages. Therefore, we present an approach that incorporates decisions of a downstream stage into an upstream model and vice versa. Our two-stage approach decomposes the problem into planning stages such as aircraft-path assignment defined in Section 4.1 and robust airline scheduling defined in Section 4.2. In each iteration, two-stage algorithm solves each subproblem by giving the output of one subproblem as an input for other subproblem. In the first subproblem, we assign aircraft types to paths for a given flight schedule with departure times, cruise times and idle times. In the second subproblem, we impose assignment decisions into the robust airline scheduling model, and then construct a flight schedule with departure times, cruise times and idle times.

We first give the steps of two-stage Algorithm 1. Algorithm 1 starts with an initial schedule $S c h_{1}$, and given cruise times $\vec{f}_{1}$ and idle times $\vec{S}_{1}$ of the schedule $S c h_{1}$. With given cruise and idle times, the algorithm applies the Construction algorithm to generate a new schedule by iteratively solving the 


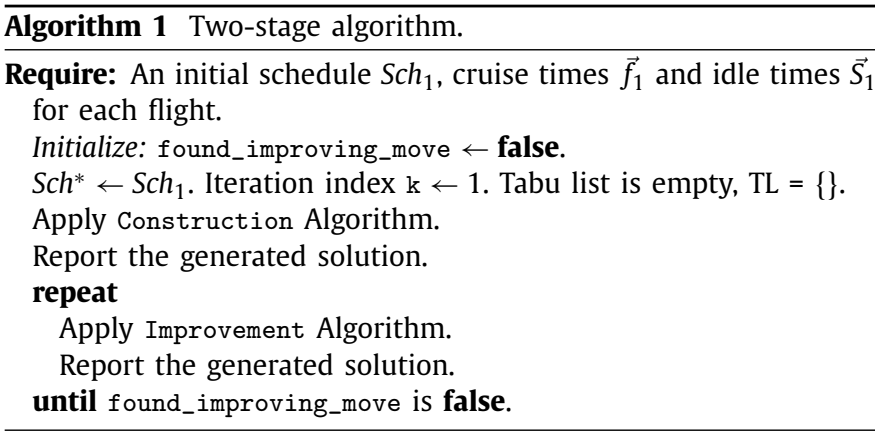

aircraft-path assignment problem defined in Section 4.1 and robust airline scheduling problem defined in Section 4.2, and then records the solution. The next step is to apply Improvement algorithm by changing the fleet assignment sequences and solving the robust airline scheduling problem again. The algorithm terminates when no further reduction in overall operational cost can be attained. In the following, we will describe two subproblems, which motivates the construction and improvement algorithms, and then we will describe the steps of Algorithm 1 in detail.

\subsection{Subproblem 1: Aircraft-Path assignment problem}

We first define aircraft-path assignment problem. The solution to the subproblem is an input for Construction algorithm. Given cruise times $\vec{f}_{k}$ and idle times $\vec{S}_{k}$ of the schedule $S_{k}$, aircraftpath assignment problem finds an optimal fleet assignment sequence $\vec{z}_{k}$ to flight paths without assigning an aircraft more than once such that all paths are covered. The objective is to minimize the overall operational cost of the schedule. The problem is formulated as follows:

$$
\begin{aligned}
& A A M(\vec{f}, \vec{S}): \min \sum_{p \in P} \sum_{t \in T} T C_{p}^{t}(\vec{f}, \vec{S}) \cdot z_{p}^{t} \\
& \text { s.to } \quad \sum_{t \in T} z_{p}^{t}=1 \quad p \in P \\
& \sum_{p \in P} z_{p}^{t} \leq N^{t} \quad t \in T \\
& z_{p}^{t} \in\{0,1\} \quad p \in P, t \in T
\end{aligned}
$$

where the total cost $T C_{p}^{t}(\vec{f}, \vec{S})$ involving the costs of fuel consumption and $\mathrm{CO}_{2}$ emission, cost of spilled passengers and cost of idle time for each path $p \in P$ and for each aircraft type $t \in T$ is calculated as follows:

$$
\begin{aligned}
& T C_{p}^{t}(\vec{f}, \vec{S})=\sum_{i \in J_{p}}\left(c_{\text {fuel }}+c_{\mathrm{CO}_{2}} \cdot k\right) \\
& \cdot\left(c_{1}^{i, t} \cdot \frac{1}{\vec{f}_{i}^{t}}+c_{2}^{i, t} \cdot \frac{1}{\left(\vec{f}_{i}^{t}\right)^{2}}+c_{3}^{i, t} \cdot\left(\vec{f}_{i}^{t}\right)^{3}+c_{4}^{i, t} \cdot\left(\vec{f}_{i}^{t}\right)^{2}\right) \\
& +\sum_{i \in J_{p}} \operatorname{Csp}_{i} \cdot \max \left(D_{i}-C A P^{t}, 0\right)+\sum_{i \in J_{p}} \vec{S}_{i}^{t} \cdot I^{t}
\end{aligned}
$$

\subsection{Subproblem 2: Robust airline scheduling problem}

We define another subproblem robust airline scheduling problem. The solution of this subproblem is an input for both Construction and Improvement algorithms. Given a fleet assignment sequence $\vec{z}_{k}$ to paths, robust airline scheduling problem finds optimal cruise times $\overrightarrow{f_{k}}$ and idle times $\overrightarrow{S_{k}}$ while ensuring the desired passenger connection service level. We achieve robustness by ensuring passenger connections at a desired service level via introducing time windows on departures and controlling cruise speed, by leaving the fleeting decisions unchanged. The objective is to minimize fuel consumption and $\mathrm{CO}_{2}$ emission costs and idle times cost. The problem is formulated as follows:

$$
\begin{aligned}
& \operatorname{RASM}(\vec{z}): \min \sum_{p \in P} \sum_{i \in J_{p}} \sum_{t \in T} C_{\text {fuel\&CO }}^{i, t}\left(f_{i}^{t}\right) \\
& \quad+\sum_{p \in P} \sum_{i \in J_{p}} \sum_{t \in T} \operatorname{Csp}_{i} \cdot \max \left(D_{i}-C A P^{t}, 0\right) \cdot \vec{z}_{p}^{t} \\
& \quad+\sum_{i \in J} \sum_{t \in T} S_{i}^{t} \cdot I_{i}^{t} \\
& \text { s.to } \quad \sum_{i \in J} \sum_{j \in P_{i}} w_{i j} \cdot \gamma_{i j} \geq \gamma
\end{aligned}
$$$$
(5)-(12)
$$

$\operatorname{RASM}(\vec{z})$ is a nonlinear model of probabilistic constraints and nonlinear cost components. Exact and fast solutions are obtained by the use of second order conic programming reformulations as discussed in the previous section.

\subsection{Construction algorithm}

Algorithm 2 gives the Construction algorithm. The algorithm starts with an initial schedule and given cruise times and idle times. Afterwards, it solves Aircraft-Path Assignment subproblem to find an optimal fleet assignment sequence to minimize the overall operational cost. The optimal fleet assignment sequence is imposed upon the Robust Airline Scheduling subproblem to find the optimal cruise times and idle times corresponding the optimal fleet assignment sequence. This procedure iteratively continues to obtain schedules with improved operational cost. The algorithm terminates when it is stuck in the same fleet assignment sequence.

In the Construction algorithm, there may exist some incompatibilities between two subproblems, which arise as an inevitable consequence of sequential planning approach. For instance, schedule generated by the RASM may not be feasible for aircraft connections of the subsequent fleet assignment. The main reason of this infeasibility is that the RASM ensures aircraft connections by considering turnaround time requirement for only the fleet assignment solution of the previous AAM. Any fleet assignment different than the previous one may require longer turnaround times, thus

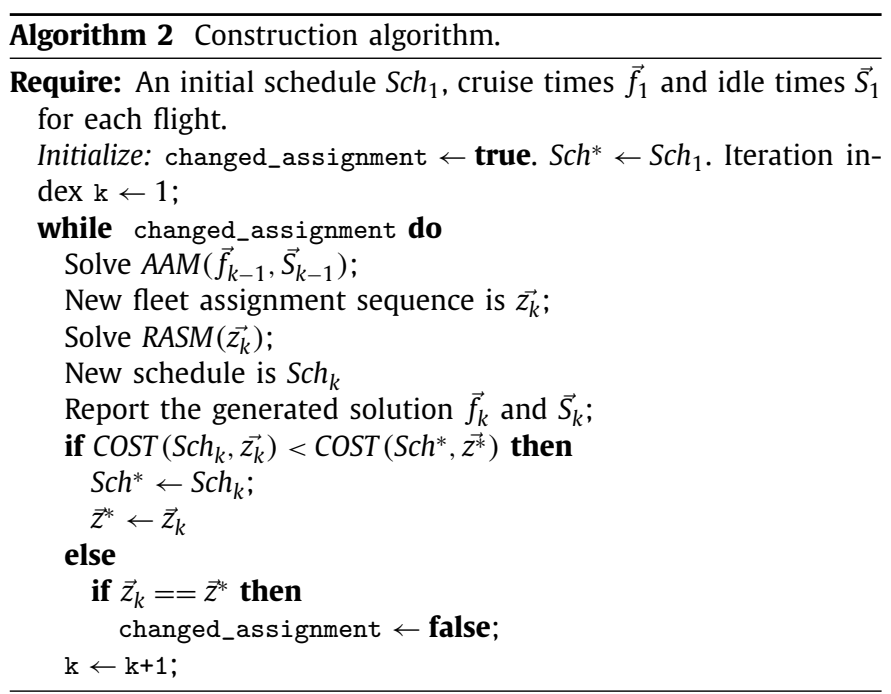


resulting in aircraft misconnections. To decrease these incompatibilities, we resolve the RASM to generate a new feasible solution by taking the fleet assignment, for which the previous schedule is infeasible, as an input. When the AAM model produces the same fleet assignment as the best one found so far, Construction algorithm terminates. This could be a local solution for the problem. To explore different fleet assignment sequences, in the next section, we describe a neighbourhood search procedure which is used by an Improvement algorithm.

\subsection{All pairwise interchange}

To explore a larger solution space, one of the most common approaches in the scheduling literature is the pairwise interchange method. This method compares the cost of two fleet assignment sequences which differ only by interchanging a pair of fleet with two different types. We will explain how to generate many fleet assignment sequences from one fleet assignment sequence with a small example. Let the best fleet type assignment sequence for four paths be $\overrightarrow{z^{*}}:=(1,2,2,3)$ where numbers 1,2 and 3 correspond to the fleet types. All Pairwise Interchange Method produces the following five fleet type assignment sequences; $\vec{z}_{1}=(2,1,2,3), \vec{z}_{2}$ $=(2,2,1,3), \vec{z}_{3}=(3,2,2,1), \vec{z}_{4}=(1,3,2,2), \vec{z}_{5}=(1,2,3,2)$. For example, the first assignment sequence, $z_{1}$ is constructed by interchanging fleet 1 and 2 between 1 st and 2 nd paths.

We generate many fleet assignment sequences at each iteration of the Improvement algorithm. The question is which fleet type assignment sequence will be selected at each iteration. The rule is to select the one with the smallest overall operational cost. The costs of the schedule $S^{*}$ with many different fleet type assignment sequences are calculated using formula (41).

\subsection{Improvement algorithm}

Algorithm 3 gives the Improvement algorithm. The algorithm starts with the schedule which is generated by Construction algorithm and iteratively applies All Pairwise Interchange to generate a neighbourhood involving different fleet assignment sequences. Algorithm 3 selects the most promising fleet assignment sequence by solving RAS problem and comparing the cost of corresponding schedules. Therefore, a schedule with improved cost is obtained at each iteration of the algorithm. A reverse move of the improving move of the fleet assignment sequence $\vec{z}^{*} \leftarrow \vec{z}_{i^{*}}$ is added to the top of the tabu list to prevent cycling. If tabu list is longer than $L$, the last entry is removed from the tabu list. Algorithm terminates when no improvement is possible for the current schedule.

In this section, we have described a heuristic algorithm which decomposes the problem into two planning stages, such as aircraft assignment and robust airline scheduling, and then solves them sequentially. Unfortunately, a sequential approach eliminates the interdependencies, thus resulting in a suboptimal solution or even infeasible solution. To reduce incompatibilities between subproblems, decisions of the schedule design problem are imposed upon the aircraft assignment model, whereas decisions of aircraft-path assignment problem are imposed upon the robust airline scheduling model in each iteration. In the next section, using data of a major U.S. airline, we show that our two-stage approach is both tractable and capable of producing very promising results to the aircraft-path assignment and robust airline scheduling problem.

\section{Computational results}

In our computational study, we test the performance of mixed integer second order conic programming formulation introduced in Section 3 and two-stage algorithm introduced in Section 4. We

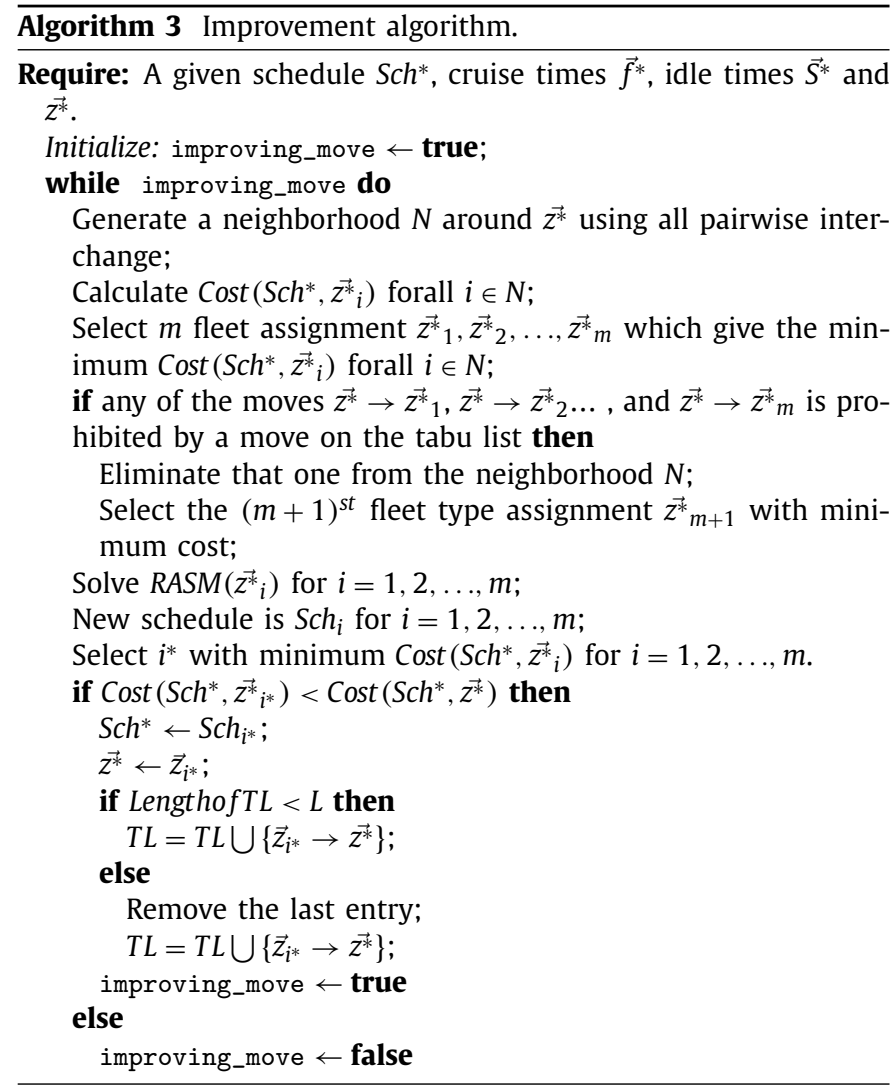

Table 3

Factor values.

\begin{tabular}{lll}
\hline & Levels & \\
\hline Factor description & Low & High \\
\hline$C_{\text {fuel }}(\$ / \mathrm{kg})$ & 0.6 & 1.2 \\
Base Spill Cost $(\$ /$ passenger $)$ & 15 & 60 \\
$\beta$ & 0.01 & 0.05 \\
\hline
\end{tabular}

test these two approaches on three different schedules generated from the work of Aktürk et al. (2014). The flight information is extracted from database "Airline On-Time Performance Data," provided by the Bureau of Transportation Statistics of the US Department of Transportation, BTS (2010a). We perform experiments in Sections 5.1 and 5.2 on a 64-bit Windows 7 computer with 8 GB memory and Intel Xeon E5640 2.67 GHz CPU. We implement the conic quadratic mixed integer reformulation and the two-stage algorithm in JAVA programming language with a connection to commercial solver IBM ILOG CPLEX Optimization Studio 12.5. Then, we perform the experiments in Section 5.3 on a OS X Yosemite computer with $8 \mathrm{~GB}$ memory and 2,6 GHz Intel Core i5 processor and use commercial solver IBM ILOG CPLEX Optimization Studio 12.6.

In order to analyze the effects of problem parameters, we conduct a $2^{k}$ full-factorial experimental design. The experimental factors are chosen and their levels are given in Table 3 .

$C_{\text {fuel }}$ is the price of jet fuel per kg. History of fuel prices obtained from IATA fuel price monitor IATA (2014) indicates that price of one kilogram fuel is fluctuating between $\$ 0.5$ and $\$ 1.26$ in 2015 . In this study, fuel prices are taken as $\$ 0.6 / \mathrm{kg}$ and $\$ 1.2 / \mathrm{kg}$ for lower and higher settings, respectively.

Base Spill Cost represents the opportunity cost for each of spilled passengers due to the insufficient seat capacity of the aircraft. Number of spilled passengers is directly affected by the fleet assignment decisions. In order to assess the overall impact of the 
number of spilled passengers on the airline's operating cost as depicted as $F 1$ in (1) in the proposed formulation, we have selected the base spill cost as an experimental factor in our computational settings and its corresponding low and high levels as shown in Table 3. Afterwards, the cost of spilled passengers is adjusted for each flight using airport congestion coefficients, e.g. favoring the populated markets, as shown in Eq. (44).

$\operatorname{Csp}_{i}=$ BaseSpillCost $\cdot\left(e_{O_{i}}\right) \cdot\left(e_{\mathrm{Dn}_{i}}\right)$

The third experimental factor, $\beta$, is used for the tail parameter of Log-Laplace distribution. Duran et al. (2015) state that the highest possible value of $\beta$ is 0.07 to ensure that tail parameter $\beta_{i}>$ 1 for each flight $i \in J$ needed to have finite expected values. Therefore, we analyze the performance of the schedule by setting $\beta$ values as 0.01 and 0.05 under the low and high settings, respectively. With a scale parameter, $e^{\alpha}$, the tail parameter is used to expect the mean and variance of the random variable. In this study, the scale parameter, $e^{\alpha}$ is taken as 20 to have a non-cruise time deviating from $20 \mathrm{~min}$.

To be used for computational purposes, we construct a sample of flight schedules in Table 4 from the work of Aktürk et al. (2014). We queried the planned departure and arrival times of a commercial airline flights for a single day. Then, we filtered the schedule such that an aircraft first departs from Chicago O'Hare International Airport (ORD) and revisits ORD at least once on the same day. This allows us to work on a schedule of flights, which has passenger connections at such congested airports. The schedule has 114 flights operated by 32 different aircraft. In this study, to observe the effect of seat capacity and fuel efficiency of the aircraft on the fleet assignment, we consider randomly selected six different aircraft types. A group of flights under the same tail number represents an aircraft path operated by the same aircraft. For the computational study, we adopt the fuel burn model of BADA (EUROCONTROL, 2012) mentioned in Section 2.1.3. The fuel consumption coefficients of an aircraft are taken from operational performance files provided by BADA (EUROCONTROL, 2012). For six different aircraft types, we list the fuel burn related parameters, corresponding MRC speed and seat capacity in Table 5. Base turn times of aircraft are adopted from airplane characteristics of Boeing company Boeing. We have used the initial investment cost of each aircraft type to estimate the idle time cost of each aircraft type in dollars per minute as summarized in Table 5 .

We generate passenger demand uniformly between 110 and 134, 110 and 122, 110 and 148, 150 and 172, 160 and 180, 160 and 218 , if originally assigned aircraft types in the published schedule are B727 228, B737 500, MD 83, A320 111, A320 212 and B767 300, respectively. We can assume that the original aircraft assignment satisfies all passenger demand. Under this experimental setting, we can clearly analyze the performance of aircraft assignment considering the fuel efficiency of the aircraft as well as the passenger demand compared to an assignment decision solely focusing on the passenger demand satisfaction.

In the computational study, we compare the schedule generated by our model to the published schedule in Table 4 . We make some assumptions on the published schedule, such that 20 min of the flight times in the published schedule in Table 4 are taken as planned non-cruise times and remaining is planned cruise time. For example, planned flight time for flight 2303 is $155 \mathrm{~min}$. 135 min of the flight time are assumed to be the planned cruise times and $20 \mathrm{~min}$ are planned non-cruise times in the published schedule. In our model, we represent the non-cruise times with a random variable which has a log-Laplace distribution. To be consistent with the published schedule, we take the scale parameter, $e^{\alpha}$ of the random variable as 20 to have a non-cruise time deviating from $20 \mathrm{~min}$. We calculate the cruise length by multiplying MRC speed of originally assigned fleet type with planned cruise

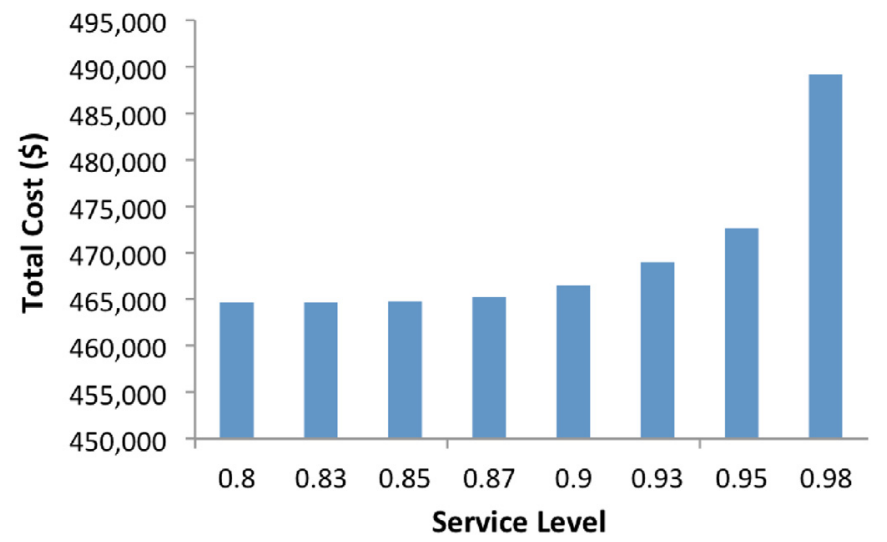

Fig. 3. What if Analysis on the Service Level.

time under the assumption that speed is constant during the cruise stage. We also calculate the upper bound $f_{i}^{u, t}$ for cruise times by dividing the cruise length of flight, $i$ with MRC speed of the aircraft, $t$. In our model, we allow compression on cruise times by $15 \%$ of $f_{i}^{u, t}$.

We add a generated passenger connection network to the published schedule. We construct a set $P_{i}$ to represent flights which have a passenger connection with flight $i$. We include flight $j$ in set $P_{i}$, if the original departure time of flight $j$ in the published schedule is within $45 \mathrm{~min}$ or $180 \mathrm{~min}$ of the original arrival time of flight $i$ and destination airport of flight $i$ is same as the origin airport of flight $j$. Passenger connection times, $T P_{i j}$ in constraint 5 are taken uniformly between 25 and $40 \mathrm{~min}$. The weights of the passenger connections, $w_{i j}$ are assigned by adjusting the number of passengers connected. Turn back in one way is not allowed for the passenger connections.

In Table 6, we give the airport congestion coefficients used in this experimental study. These coefficients are normalized using the number of passengers visiting the airports, which is obtained from the T-100 market data of BTS (2010b). The most congested airport in the published schedule has 1.96 congestion level and the least congested airport has 0.64 .

We estimate the aircraft turnaround time needed for an aircraft at an airport by multiplying the base aircraft turntime with the airport congestion coefficients as in Eq. (45). Therefore, turntime of an aircraft visiting a congested airport is longer. The calculated aircraft turntimes match with the aircraft turntimes given in Arıkan et al. (2012). Moreover, turntime between through flights are taken as $70 \%$ of the calculated turntimes, because boarding time of passengers and time to load and unload their cargos require less time.

$T A_{i j}^{t}=$ BaseTurntime $^{t} \cdot e_{\mathrm{Dn}_{i}}$

To be consistent with the published schedule, the departure time of the first flight of each path is set to the departure time of the first flight in the published schedule. Time window constraints are imposed upon the rest of the flights.

In this study, we have a bi-criteria problem where the conflicting objectives are maximization of the service level for passenger connections and minimization of airline's overall cost. In Fig. 3, we show the efficient frontier for a single problem instance using a flight schedule with the first 41 flights of the published schedule in Table 4. We set each experimental factor to the highest value. It can be clearly seen that, when airlines try to maximize the service level for passenger connections, their operational cost increases as expected. The sharp increase in the operational cost occurs while ensuring a higher service level than 0.95 . On the other hand, the minimum cost is obtained at the lowest service level. 
Table 4

Published schedule.

\begin{tabular}{|c|c|c|c|c|c|c|c|c|c|c|c|c|c|}
\hline Tail No & Flight no & Departure & Arrival & $\begin{array}{l}\text { Departure } \\
\text { time }\end{array}$ & Flight time & Arrival time & Tail no & Flight No & Departure & Arrival & $\begin{array}{l}\text { Departure } \\
\text { time }\end{array}$ & Flight time & Arrival time \\
\hline N531AA & 2303 & ORD & DFW & $6: 45$ & $2: 35$ & 9:20 & N3DMAA & 568 & ORD & FLL & $7: 25$ & $2: 55$ & $10: 20$ \\
\hline N531AA & 2336 & DFW & ORD & $10: 10$ & $2: 30$ & $12: 40$ & N3DMAA & 711 & FLL & ORD & $11: 10$ & $3: 15$ & $14: 25$ \\
\hline N531AA & 1053 & ORD & AUS & $13: 25$ & $2: 50$ & $16: 15$ & N3DMAA & 2021 & ORD & SJU & $15: 25$ & $4: 35$ & 20:00 \\
\hline N531AA & 336 & AUS & ORD & $17: 00$ & $2: 45$ & $19: 45$ & N544AA & 2463 & ORD & $\mathrm{MCI}$ & $6: 25$ & $1: 30$ & $7: 55$ \\
\hline N531AA & 336 & ORD & LGA & $20: 40$ & $2: 14$ & $22: 54$ & N544AA & 754 & $\mathrm{MCI}$ & ORD & $8: 40$ & $1: 30$ & $10: 10$ \\
\hline N598AA & 1341 & ORD & SFO & 7:50 & 4:55 & $12: 45$ & N544AA & 2321 & ORD & DFW & $11: 15$ & $2: 35$ & $13: 50$ \\
\hline N598AA & 348 & SFO & ORD & $13: 30$ & $4: 25$ & $17: 55$ & N544AA & 2356 & DFW & ORD & $14: 40$ & $2: 30$ & $17: 10$ \\
\hline N598AA & 1521 & ORD & TUS & 19:15 & 3:55 & 23:10 & N544AA & 2487 & ORD & DEN & $17: 50$ & $2: 45$ & $20: 35$ \\
\hline N475AA & 407 & ORD & STL & $6: 20$ & $1: 10$ & $7: 30$ & N3EBAA & 1565 & ORD & MSP & $6: 40$ & $1: 30$ & $8: 10$ \\
\hline N475AA & 755 & STL & ORD & $8: 35$ & $1: 15$ & 9:50 & N3EBAA & 779 & MSP & ORD & 9:00 & $1: 25$ & $10: 25$ \\
\hline N475AA & 755 & ORD & SAT & $10: 45$ & 3:00 & $13: 45$ & N3EBAA & 779 & ORD & SAN & $11: 35$ & $4: 20$ & $15: 55$ \\
\hline N475AA & 408 & SAT & ORD & $14: 30$ & $2: 40$ & $17: 10$ & N3EBAA & 1358 & SAN & ORD & $16: 45$ & $3: 55$ & $20: 40$ \\
\hline N475AA & 408 & ORD & PHL & 18:05 & 2:05 & $20: 10$ & N3EBAA & 1358 & ORD & BOS & $21: 50$ & $2: 10$ & $0: 00$ \\
\hline N3EEAA & 876 & ORD & BOS & $6: 35$ & $2: 10$ & $8: 45$ & N3ETAA & 1704 & ORD & EWR & $6: 35$ & 2:05 & $8: 40$ \\
\hline N3EEAA & 413 & BOS & ORD & 9:35 & 3:05 & $12: 40$ & N3ETAA & 1883 & EWR & ORD & 9:30 & $2: 40$ & $12: 10$ \\
\hline N3EEAA & 413 & ORD & SNA & $13: 45$ & $4: 35$ & $18: 20$ & N3ETAA & 810 & ORD & DCA & $13: 10$ & $1: 40$ & $14: 50$ \\
\hline N3EEAA & 1262 & SNA & ORD & 19:10 & 3:50 & 23:00 & N3ETAA & 2013 & DCA & ORD & $15: 45$ & $2: 10$ & $17: 55$ \\
\hline N4YDAA & 451 & ORD & SFO & $9: 45$ & $4: 55$ & $14: 40$ & N3ETAA & 2013 & ORD & LAS & $19: 00$ & 4:05 & 23:05 \\
\hline N4YDAA & 554 & SFO & ORD & $15: 45$ & $4: 25$ & $20: 10$ & N3DYAA & 1063 & ORD & LAX & $8: 50$ & $4: 35$ & $13: 25$ \\
\hline N3ERAA & 496 & ORD & DCA & $6: 45$ & $1: 40$ & $8: 25$ & N3DYAA & 874 & LAX & ORD & $14: 30$ & $4: 15$ & $18: 45$ \\
\hline N3ERAA & 1715 & DCA & ORD & 9:15 & $2: 10$ & $11: 25$ & N3DYAA & 874 & ORD & BOS & $19: 45$ & $2: 10$ & 21:55 \\
\hline N3ERAA & 1715 & ORD & LAS & $12: 25$ & 4:05 & $16: 30$ & N5DXAA & 1048 & ORD & MIA & $7: 35$ & $3: 00$ & $10: 35$ \\
\hline N3ERAA & 1708 & LAS & ORD & $17: 20$ & $3: 40$ & 21:00 & N5DXAA & 1763 & MIA & ORD & $11: 55$ & $3: 20$ & $15: 15$ \\
\hline N5CLAA & 1425 & ORD & SNA & $8: 25$ & $4: 40$ & 13:05 & N5DXAA & 1899 & ORD & MIA & $16: 20$ & 3:00 & 19:20 \\
\hline N5CLAA & 556 & SNA & ORD & $14: 00$ & 4:00 & 18:00 & N454AA & 2441 & ORD & ATL & $6: 30$ & $2: 00$ & $8: 30$ \\
\hline N5CLAA & 1940 & ORD & MIA & $19: 25$ & 3:00 & $22: 25$ & N454AA & 1986 & ATL & ORD & $9: 15$ & $2: 15$ & $11: 30$ \\
\hline N535AA & 2460 & ORD & RSW & $6: 45$ & $2: 45$ & 9:30 & N454AA & 1872 & ORD & MCO & $12: 25$ & $2: 40$ & $15: 05$ \\
\hline N535AA & 564 & RSW & ORD & $10: 20$ & 3:05 & $13: 25$ & N454AA & 1131 & MCO & ORD & $15: 50$ & 3:05 & $18: 55$ \\
\hline N535AA & 1446 & ORD & EWR & $14: 55$ & $2: 45$ & $17: 40$ & N4YMAA & 1137 & ORD & MSY & $8: 20$ & $2: 25$ & $10: 45$ \\
\hline N535AA & 1411 & EWR & ORD & $18: 45$ & $2: 45$ & $21: 30$ & N4YMAA & 1768 & MSY & ORD & $11: 30$ & $2: 30$ & $14: 00$ \\
\hline N3DRAA & 1021 & ORD & LAS & $8: 30$ & 4:05 & $12: 35$ & N4YMAA & 1768 & ORD & PHL & $15: 05$ & 2:05 & $17: 10$ \\
\hline N3DRAA & 1544 & LAS & ORD & $13: 25$ & $3: 40$ & $17: 05$ & N4YMAA & 1697 & PHL & ORD & $18: 00$ & $2: 35$ & $20: 35$ \\
\hline N3DRAA & 1544 & ORD & DCA & 18:00 & $1: 40$ & $19: 40$ & N420AA & 1686 & ORD & RDU & $6: 50$ & $1: 50$ & $8: 40$ \\
\hline N467AA & 1823 & ORD & PBI & $9: 20$ & $2: 55$ & $12: 15$ & N420AA & 2435 & RDU & ORD & $9: 25$ & $2: 15$ & $11: 40$ \\
\hline N467AA & 2067 & PBI & ORD & 13:00 & $3: 20$ & $16: 20$ & N420AA & 2435 & ORD & PHX & $12: 35$ & 4:00 & $16: 35$ \\
\hline N467AA & 2067 & ORD & STL & $17: 15$ & $1: 10$ & $18: 25$ & N420AA & 1206 & PHX & ORD & $17: 15$ & $3: 30$ & $20: 45$ \\
\hline N467AA & 1186 & STL & ORD & 19:10 & $1: 15$ & $20: 25$ & N546AA & 1462 & ORD & EWR & 8:00 & $2: 45$ & $10: 45$ \\
\hline N3DTAA & 2363 & ORD & HDN & $9: 50$ & $2: 50$ & $12: 40$ & N546AA & 1387 & EWR & ORD & $11: 25$ & $2: 45$ & $14: 10$ \\
\hline N3DTAA & 2318 & HDN & ORD & $13: 40$ & $2: 50$ & $16: 30$ & N546AA & 1397 & ORD & MCO & $15: 00$ & $2: 40$ & $17: 40$ \\
\hline N412AA & 2345 & ORD & DFW & 17:15 & $2: 35$ & 19:50 & N546AA & 1221 & MCO & ORD & $18: 25$ & $2: 55$ & $21: 20$ \\
\hline N412AA & 2374 & DFW & ORD & $20: 40$ & $2: 20$ & 23:00 & N4WPAA & 2311 & ORD & DFW & 9:05 & $2: 35$ & $11: 40$ \\
\hline N530AA & 398 & ORD & LGA & $6: 15$ & 2:14 & $8: 29$ & N4WPAA & 2348 & DFW & ORD & $12: 35$ & $2: 20$ & $14: 55$ \\
\hline N530AA & 319 & LGA & ORD & $9: 25$ & $2: 50$ & $12: 15$ & N4WPAA & 1797 & ORD & STL & $15: 50$ & $1: 10$ & $17: 00$ \\
\hline
\end{tabular}


Table 4 (continued)

\begin{tabular}{|c|c|c|c|c|c|c|c|c|c|c|c|c|c|}
\hline Tail No & Flight no & Departure & Arrival & $\begin{array}{l}\text { Departure } \\
\text { time }\end{array}$ & Flight time & Arrival time & Tail no & Flight No & Departure & Arrival & $\begin{array}{l}\text { Departure } \\
\text { time }\end{array}$ & Flight time & Arrival time \\
\hline N530AA & 2329 & ORD & DFW & 13:35 & $2: 35$ & $16: 10$ & N4WPAA & 1982 & STL & ORD & $18: 00$ & $1: 15$ & 19:15 \\
\hline N530AA & 2364 & DFW & ORD & 17:00 & $2: 30$ & $19: 30$ & N4WPAA & 1339 & ORD & SAN & $20: 15$ & $4: 30$ & $0: 45$ \\
\hline N459AA & 394 & ORD & LGA & $6: 50$ & $2: 15$ & 9:05 & N439AA & 2455 & ORD & PHX & $7: 10$ & 4:00 & $11: 10$ \\
\hline N459AA & 321 & LGA & ORD & $10: 00$ & $2: 50$ & $12: 50$ & N439AA & 358 & PHX & ORD & 11:55 & $3: 30$ & $15: 25$ \\
\hline N459AA & 366 & ORD & LGA & 13:55 & $2: 20$ & $16: 15$ & N439AA & 358 & ORD & LGA & $16: 25$ & $2: 15$ & $18: 40$ \\
\hline N459AA & 347 & LGA & ORD & 17:15 & $2: 50$ & $20: 05$ & N439AA & 371 & LGA & ORD & $20: 00$ & $2: 50$ & 22:50 \\
\hline N4XGAA & 2079 & ORD & SAN & $8: 45$ & $4: 30$ & 13:15 & N5EBAA & 2375 & ORD & EGE & $8: 10$ & $2: 55$ & 11:05 \\
\hline N4XGAA & 1438 & SAN & ORD & 14:00 & 4:10 & $18: 10$ & N4EBAA & 2378 & EGE & ORD & $12: 25$ & $2: 45$ & $15: 10$ \\
\hline N4XGAA & 346 & ORD & LGA & 19:50 & $2: 15$ & 22:05 & N4EBAA & 1677 & ORD & SNA & $18: 40$ & $4: 40$ & $23: 20$ \\
\hline N536AA & 2305 & ORD & DFW & $7: 45$ & $2: 35$ & $10: 20$ & N3DUAA & 2099 & ORD & LAX & 7:00 & $4: 35$ & $11: 35$ \\
\hline N536AA & 2344 & DFW & ORD & 11:35 & $2: 30$ & 14:05 & N3DUAA & 1972 & LAX & ORD & $12: 40$ & 4:15 & $16: 55$ \\
\hline N536AA & 1201 & ORD & STL & 14:50 & 1:05 & $15: 55$ & N3DUAA & 1972 & ORD & RDU & $17: 45$ & $1: 55$ & $19: 40$ \\
\hline N536AA & 1815 & STL & ORD & 17:00 & $1: 20$ & $18: 20$ & N3ELAA & 2057 & ORD & SJU & $8: 30$ & $4: 35$ & 13:05 \\
\hline N536AA & 1815 & ORD & SLC & 19:15 & $3: 40$ & $22: 55$ & N3ELAA & 2078 & SJU & ORD & $14: 25$ & 5:35 & $20: 00$ \\
\hline
\end{tabular}


Table 5

Aircraft parameters.

\begin{tabular}{lllllll}
\hline Aircraft type & B727 228 & B737 500 & MD 83 & A320 111 & A320 212 & B767 300 \\
\hline Capacity & 134 & 122 & 148 & 172 & 180 & 218 \\
Mass $(\mathrm{kgs})$ & 74,000 & 50,000 & 61,200 & 62,000 & 64,000 & 135,000 \\
Surface $\left(m^{2}\right)$ & 157.9 & 105.4 & 118 & 122.4 & 122.6 & 283.3 \\
$C_{D 0, C R}$ & 0.018 & 0.018 & 0.0211 & 0.024 & 0.024 & 0.021 \\
$C_{D 2, C R}$ & 0.06 & 0.055 & 0.0468 & 0.0375 & 0.0375 & 0.049 \\
$C f_{1}$ & 0.53178 & 0.46 & 0.7462 & 0.94 & 0.94 & 0.763 \\
$C f_{2}$ & 276.72 & 300 & 638.59 & 50,000 & 100,000 & 1,430 \\
$c f_{C R}$ & 0.954 & 1.079 & 0.9505 & 1.095 & 1.06 & 1.0347 \\
MRC speed & 867.6 & 859.2 & 867.6 & 855.15 & 868.79 & 876.70 \\
Base turntime & 32 & 36 & 26 & 28 & 30 & 40 \\
Idle time cost $(\$)$ & 150 & 140 & 142 & 136 & 144 & 147 \\
\hline
\end{tabular}

Table 6

Congestion coefficients $\left(e_{b}\right)$.

\begin{tabular}{llllll}
\hline Airport & Location & Coefficient & Airport & Location & Coefficient \\
\hline MIA & Miami, FL & 1.96 & DCA & Washington, DC & 1.17 \\
ORD & Chicago, IL & 1.88 & SAN & San Diego, CA & 1.10 \\
LAX & Los Angeles, CA & 1.82 & STL & St.Louis, MO & 1.10 \\
DEN & Denver,CO & 1.82 & MCI & Kansas City, MO & 1.04 \\
DFW & Dallas, TX & 1.74 & AUS & Austin, TX & 1.00 \\
LGA & New York, NY & 1.69 & RDU & Raleigh/Durham, NC & 1.00 \\
BOS & Boston, MA & 1.69 & MSY & New Orleans, LA & 0.96 \\
ATL & Atlanta, GA & 1.64 & SNA & Santa Ano, CA & 0.96 \\
PHX & Phoenix, AZ & 1.56 & SAT & San Antonio, TX & 0.90 \\
LAS & Las Vegas, NV & 1.56 & RSW & Fort Myers, FL & 0.90 \\
SFO & San Fransisco, CA & 1.44 & SJU & San Juan, PR & 0.85 \\
MSP & Minneapolis, MN & 1.32 & PBI & West Palm Beach, FL & 0.81 \\
PHL & Philadelphia, PA & 1.32 & TUS & Tuscan, AZ & 0.77 \\
EWR & Newark, NJ & 1.25 & MCO & Orlando, FL & 0.72 \\
FLL & Fort Lauderdale, FL & 1.25 & EGE & Eagle, CO & 0.72 \\
SLC & Salt Lake City, UT & 1.17 & HDN & Hayden, CO & 0.64 \\
\hline
\end{tabular}

Table 7

Original aircraft types.

\begin{tabular}{llll}
\hline Tail no & Aircraft type & Tail no & Aircraft type \\
\hline N531AA & B767 300 & N5CLAA & B767 300 \\
N598AA & B767 300 & N535AA & B767 300 \\
N475AA & A320 212 & N3DRAA & A320 111 \\
N3EEAA & A320 111 & N467AA & A320 212 \\
N4YDAA & A320 212 & N3DTAA & A320 111 \\
N3ERAA & A320 111 & N412AA & A320 212 \\
\hline
\end{tabular}

\subsection{Analysis on the schedule with 41 flights}

In this experimental study, we use a flight schedule which is generated by taking the first 41 flights of the published schedule in Table 4. We consider 12 different aircraft with 3 different types and randomly assign aircraft types in Table 7.

We first test the performance of the schedule achieved by the integrated model with the published schedule. Afterwards, we compare the results of the two-stage algorithm and the optimal solutions of the integrated model.

\subsubsection{Computational analysis on the integrated model}

We give a comparison among different cost components between the integrated model's optimum solution and published schedule in Table 8 . We summarize the results for 40 problem instances. For each factor level, we report the minimum, average and maximum reduction in fuel and emission costs, idle time cost, total cost and total cost without considering delay over five replications. We consider delay cost of the published schedule, when we calculate the total cost improvement. Unit delay cost is difficult to determine. To simplify the calculation, we assume a linear delay cost with $\$ 200$ per minute of delay. But, it is evident that integrated model performs better cost savings in total cost even without including the delay cost. The improvement percentages are calculated using the following formula:

$$
\begin{aligned}
& \text { Cost Improvement }(\%) \\
& =100 \times \frac{\text { Published Schedule - Integrated Model }}{\text { Published Schedule }}
\end{aligned}
$$

The effect of the fuel price per ton has a significant impact on the total cost improvement. The integrated model has much more tendency to decrease fuel consumption, when fuel price is high. Therefore, integrated model assigns the fuel efficient aircraft to the paths which require more fuel consumption and adjusts the speed of the aircraft to minimize the fuel consumption. As a result, fuel and $\mathrm{CO}_{2}$ emission cost improvements increase. On the other hand, the cost of the spilled passengers increases with this assignment so that it leads to low total cost improvements.

When base spill cost is high, this leads to much more demand satisfaction to reduce the cost of spilled passengers. However, this approach incurs higher fuel and $\mathrm{CO}_{2}$ emission costs which constitute the major portion of the airline operational costs. Therefore, total cost saving decreases compared to the low setting.

$\beta$ scale parameter of the log-Laplace distribution has significant effects on the idle time and total cost improvements. It is observed that our model achieves better idle time and total cost savings when $\beta$ is low, and the performance of the model in improving fuel and emission costs is slightly affected by $\beta$. The reason behind this is that a higher $\beta$ causes a higher variance in non-cruise times of flights, which necessitates more idle time insertion and compression on the cruise times to ensure the connections. Therefore, this leads to more idle time and total operational costs.

Overall, improvements in the cost of fuel and $\mathrm{CO}_{2}$ emissions are approximately $11 \%$, whereas the improvement in idle time is approximately $68 \%$. However, $2 \%$ of the passengers are not satisfied due to limited seat capacity of the aircraft. It is observed that 
Table 8

Comparison of factor effects.

\begin{tabular}{|c|c|c|c|c|c|c|c|c|c|c|c|c|c|}
\hline & & \multicolumn{3}{|c|}{$\begin{array}{l}\text { Fuel \& emis. cost } \\
\text { improvement }\end{array}$} & \multicolumn{3}{|c|}{$\begin{array}{l}\text { Idle cost } \\
\text { improvement }\end{array}$} & \multicolumn{3}{|c|}{$\begin{array}{l}\text { Total cost } \\
\text { improvement }\end{array}$} & \multicolumn{3}{|c|}{$\begin{array}{l}\text { Total impr. } \\
\text { without delay }\end{array}$} \\
\hline & & Min & Avg & Max & Min & Avg & Max. & Min & Avg & Max & Min & Avg. & Max \\
\hline \multirow[t]{2}{*}{$C_{\text {fuel }}(\$ / \mathrm{kg})$} & Low & 8.3 & 11.1 & 12.3 & 59.8 & 68.4 & 79.8 & 20.5 & 24.5 & 29.2 & 18.2 & 23.3 & 28.8 \\
\hline & High & 10.5 & 11.7 & 12.3 & 59.4 & 68.1 & 79.8 & 16.6 & 19.3 & 22.4 & 15.2 & 18.5 & 22.2 \\
\hline \multirow[t]{2}{*}{ Base Spill Cost } & Low & 12.1 & 12.3 & 12.3 & 59.5 & 67.9 & 79.8 & 19.2 & 23.1 & 29.2 & 17.8 & 22.1 & 28.8 \\
\hline & High & 8.3 & 10.5 & 12.3 & 59.4 & 68.5 & 79.8 & 16.6 & 20.7 & 27.3 & 15.2 & 19.6 & 26.9 \\
\hline \multirow[t]{2}{*}{$\beta$} & Low & 8.9 & 11.6 & 12.3 & 69.2 & 75.5 & 79.8 & 17.5 & 23.2 & 29.2 & 17.2 & 22.8 & 28.8 \\
\hline & High & 8.3 & 11.1 & 12.3 & 59.4 & 61.0 & 66.3 & 16.6 & 20.7 & 24.9 & 15.2 & 18.9 & 22.7 \\
\hline
\end{tabular}

Table 9

Cost comparison for different replications.

\begin{tabular}{|c|c|c|c|c|c|c|c|c|c|}
\hline \multirow[b]{2}{*}{ Replications } & \multicolumn{3}{|c|}{$\begin{array}{l}\text { Fuel \& emis. cost } \\
\text { improvement (\%) }\end{array}$} & \multicolumn{3}{|c|}{$\begin{array}{l}\text { Idle cost } \\
\text { improvement (\%) }\end{array}$} & \multicolumn{3}{|c|}{$\begin{array}{l}\text { Total cost } \\
\text { improvement (\%) }\end{array}$} \\
\hline & Min & Avg & Max & Min & Avg & Max. & Min & Avg & Max \\
\hline 1 & 8.3 & 11.1 & 12.3 & 59.6 & 68.3 & 77.1 & 16.7 & 21.7 & 27.5 \\
\hline 2 & 8.9 & 11.1 & 12.3 & 59.4 & 67.9 & 76.3 & 16.6 & 21.5 & 27.1 \\
\hline 3 & 11.1 & 11.9 & 12.3 & 60.1 & 69.7 & 79.8 & 18.6 & 23.3 & 29.2 \\
\hline 4 & 9.0 & 11.6 & 12.3 & 61.7 & 65.9 & 70.2 & 16.7 & 20.8 & 25.8 \\
\hline 5 & 8.8 & 11.2 & 12.3 & 61.0 & 69.3 & 76.5 & 17.6 & 22.3 & 27.5 \\
\hline
\end{tabular}

Table 10

CPU time analysis of the integrated model.

\begin{tabular}{lllll}
\hline & & \multicolumn{3}{l}{ CPU time $(\mathrm{sec})$} \\
\cline { 3 - 5 } Factor & Level & Min & Avg & Max \\
\hline$C_{\text {fuel }}(\$ / \mathrm{kg})$ & Low & 758 & 2250 & 6490 \\
\multirow{3}{*}{ Base Spill Cost } & High & 606 & 3425 & 11290 \\
& Low & 606 & 3474 & 11290 \\
$\beta$ & High & 743 & 2201 & 6490 \\
& Low & 606 & 2350 & 7303 \\
& High & 743 & 3325 & 11290
\end{tabular}

the fuel and $\mathrm{CO}_{2}$ emission cost savings compensate the cost of the spilled passengers, thus resulting in $22 \%$ total cost improvement.

We take five replications for each factor combination to observe if random values of passenger demand and passenger connection times have any impact on objective values. For each replication, the minimum, average and maximum improvements in each cost component are seen in Table 9. We could conclude that there is no statistically significant randomization effect on the objective function values.

To see the effect of each factor on the computation time, minimum, average and maximum values of CPU in seconds over five replications are analyzed in Table 10 . Increase in fuel price makes aircraft-path assignment decisions more crucial. As expected this requires much more $\mathrm{CPU}$ time to deal with the nonlinear fuel and emission cost functions. If the base spill cost increases, then the seat capacity of the aircraft becomes more significant on the fleet assignment strategy. The problem complexity decreases, thereby decreases the overall computation time. Increasing the variability, i.e., $\beta$, increases the problem complexity as it becomes harder to satisfy the connections at a desired service level. As a result, the required CPU time increases.

Moreover the average CPU time requirement for solving the mixed integer second order conic formulation is $3000 \mathrm{CPU}$ seconds. As expected, the conflicting objectives increase the problem complexity and the computation time. When the fuel price and $\beta$ parameters are high and base spill cost is low, maximum time to solve the five problem instances reaches to $11,290 \mathrm{CPU}$ seconds. Therefore, we also devise a two-stage algorithm to solve the large scale problems in a reasonable time.
5.1.2. Computational analysis on the two-Stage algorithm

In this section, we use the same generated schedule having 41 flights operated by 12 different aircraft with 3 different types. We similarly perform a $2^{3}$ full-factorial experimental design with 5 replications. The parameter, $m$, to generate $m$ schedules in the twostage algorithm is set to three and the length of the tabu list is taken as 1 . In order to test the solution quality of the two-stage algorithm, we compare the total cost of the schedule generated by the two-stage algorithm with the optimum solution of the integrated model. Optimality gap for the two-stage algorithm is calculated as the following:

Optimality gap $=\frac{\text { Two-Stage Algorithm - Integrated Model }}{\text { Integrated Model }}$

For each factor combination, we provide the average optimality gap over 5 replications in Table 11 . When the factors $C_{\text {fuel }}$, Base Spill Cost and $\beta$ are set to their low, high and high values, total cost of the schedule developed by the two-stage algorithm is approximately 0.001 times worse than the total cost of the schedule achieved by the integrated model as well as the high, high and low setting, respectively. For the remaining ones, achieved costs by the two-stage algorithm are approximately the same as the optimal solutions of the integrated model.

In addition, for only 6 instances out of 40 the instances, the fleet type assignment obtained by the two-stage algorithm differs from the optimal solutions of the integrated model. The gaps between the objectives are negligible for these instances. Our computational results indicate that for the reasonable size of the problem, the two-stage algorithm gives very similar results to the optimum solutions in seconds.

\subsection{Analysis on the schedule with 114 flights}

For this computational study, we utilize the published schedule in Table 4. The schedule has 114 flights operated by 32 different aircraft with six different types. We again perform a $2^{3}$ fullfactorial experimental design with 5 replications. We first solve the mixed integer second order conic programming formulation within a time limit of 9000 CPU seconds. We obtain the best known upper bound (objective) and the best lower bound (LB) at the time of the termination. Then, we also run the two-stage algorithm. We calculate the relative gap between the LB obtained by the integrated 
Table 11

Gap between two-stage algorithm and the integrated model.

\begin{tabular}{|c|c|c|c|c|c|c|c|}
\hline \multicolumn{3}{|l|}{ Factor } & \multirow{2}{*}{$\begin{array}{l}\text { Optimality } \\
\text { Gap }\end{array}$} & \multicolumn{3}{|l|}{ Factor } & \multirow{2}{*}{$\begin{array}{l}\text { Optimality } \\
\text { Gap }\end{array}$} \\
\hline$C_{\text {fuel }}(\$ / \mathrm{kg})$ & Base Spill Cost & $\beta$ & & $C_{\text {fuel }}(\$ / \mathrm{kg})$ & Base Spill Cost & $\beta$ & \\
\hline Low & Low & Low & 0.000 & High & Low & Low & 0.000 \\
\hline Low & Low & High & 0.000 & High & Low & High & 0.000 \\
\hline Low & High & Low & 0.000 & High & High & Low & 0.001 \\
\hline Low & High & High & 0.001 & High & High & High & 0.000 \\
\hline
\end{tabular}

Table 12

Factor effects on the Gap.

\begin{tabular}{llllllll}
\hline & & \multicolumn{3}{l}{$\begin{array}{l}\text { Gap with LB (\%) } \\
\text { for integrated model }\end{array}$} & \multicolumn{3}{l}{$\begin{array}{l}\text { Gap with LB (\%) } \\
\text { for two-stage alg. }\end{array}$} \\
\hline \multirow{2}{*}{ Factor } & Level & Min & Avg & Max & Min & Avg & Max \\
\hline$C_{\text {fuel }}(\$ / \mathrm{kg})$ & Low & 5.4 & 7.1 & 10.5 & 2.2 & 6.0 & 7.7 \\
& High & 4.9 & 7.8 & 10.0 & 2.6 & 6.3 & 8.1 \\
Base Spill Cost & Low & 5.9 & 8.6 & 10.5 & 4.0 & 7.1 & 8.1 \\
& High & 4.9 & 6.2 & 7.7 & 2.2 & 5.1 & 6.1 \\
$\beta$ & Low & 4.9 & 7.2 & 10.5 & 2.2 & 5.7 & 8.0 \\
& High & 5.4 & 7.7 & 10.0 & 5.0 & 6.6 & 8.1 \\
\hline
\end{tabular}

Table 13

Factor effects on relative performance.

\begin{tabular}{lllll}
\hline & & \multicolumn{3}{l}{$\begin{array}{l}\text { Relative } \\
\text { performance }(\%)\end{array}$} \\
\cline { 3 - 5 } Factor & Level & Min & Avg & Max \\
\hline \multirow{2}{*}{$C_{\text {fuel }}(\$ / \mathrm{kg})$} & Low & 0.1 & -1.0 & -4.9 \\
\multirow{3}{*}{ Base Spill Cost } & High & -0.8 & -1.3 & -3.0 \\
& Low & -0.3 & -1.4 & -4.9 \\
$\beta$ & High & 0.1 & -1.0 & -3.0 \\
& Low & -0.3 & -1.3 & -4.9 \\
& High & 0.1 & -1.0 & -3.0 \\
\hline
\end{tabular}

model within the time limit and the objective of the two-stage algorithm. In a similar way, we also calculate the relative gap with the LB for the integrated model. We give the calculations of relative gaps with the LB as follows:

Gap with LB for Two-stage Alg. (\%)

$$
=100 \times \frac{\text { Objective of Two-stage Alg. }- \text { LB }}{\text { LB }}
$$

\section{Gap with LB for Integrated Model (\%)}

$$
=100 \times \frac{\text { Objective of Integrated Model }- \text { LB }}{\text { LB }}
$$

Table 12 illustrates the minimum, average and maximum of the relative gaps over five replications both for the integrated model and the two-stage algorithm. On the average, the gap with lower bound for the integrated model is $7.43 \%$, whereas it is $6.14 \%$ for the two-stage algorithm. It is important to clarify that, on the average, the gap for the two-stage algorithm is less than the integrated model within the time limit of 9000 CPU seconds. In other words, on the average, the two-stage algorithm results in a lower cost schedule than the best incumbent solution found by the integrated model within given time limit.

Moreover, for 18 instances out of the 40 instances, the relative gap for the two-stage algorithm becomes less than $6 \%$, whereas 6 of them result in 5\% gap. However, we do not know whether the lower bound is weak or strong. Thus, the optimality gap with the lower bound is not enough to analyze the performance of twostage algorithm. We also compare the results of the two-stage algorithm to the best incumbent solutions found by the integrated model within $9000 \mathrm{CPU}$ seconds as follows:

Relative Performance (\%)

$$
=100 \times \frac{\text { Objective of Two-stage Alg. - Objective of IM }}{\text { Objective of IM }}
$$

Integrated model could not reach optimal solutions within the time limit of 9000 CPU seconds. Even, for 39 out of 40 instances, solutions of two-stage approach give lower cost than the best incumbent solutions of the integrated model. In Table 13, negative values represent a better performance for two-stage approach compared to solutions of the integrated model within the time limit. For instance, when fuel price is low, two-stage approach obtains a maximum cost saving of $4.9 \%$ relative to the best incumbent solution. On the average of 40 instances, two-stage algorithm gives $1.18 \%$ total cost saving compared to the best solutions found by the integrated model. For 20 out of 40 instances, two-stage algorithm provides $1 \%$ cost saving, where 4 of them results in $2 \%$ cost saving. On the other hand, for only 1 instance over 40 instances, two-stage algorithm gives $0.1 \%$ higher cost than the best solution of the integrated model, when factors $C_{\text {fuel }}$, Base Spill Cost and $\beta$ are at their low, high and high levels, respectively. We can conclude that twostage approach is capable of producing very promising results compared to the best solutions of the integrated model within 9000 CPU seconds.

We also provide different cost improvements for two-stage approach compared to the published schedule in Table 14. It can be seen that, the results of the two-stage algorithm are very promising as well. Idle time cost savings are approximately $70 \%$, whereas the fuel and emission cost reductions are approximately $4.5 \%$. The overall cost saving is around $21 \%$. These are similar to the analysis on the schedule with 41 flights.

Our computational results indicate that fuel and $\mathrm{CO}_{2}$ emission cost improvements increase as the fuel price increases. In contrast, percentage of the spilled passengers increases as it is seen in Table 15. Total cost saving decreases together with increase in the cost of spilled passengers and slightly decrease in idle time cost improvement.

Table 15 also illustrates that high value of the base spill cost decreases the number of spilled passengers, as expected. However, this decreases the fuel and $\mathrm{CO}_{2}$ emission cost improvements, thereby resulting in lower total cost savings.

In Table 15, we clearly observe the impact of flight time uncertainty on the passenger spill. Table 14 shows that increase in the variability decreases the idle time cost improvements as well as fuel and carbon emission cost improvements. The reason behind this is that our approach inserts larger idle times over critical connections and compresses the cruise time of flights to ensure the passenger connections with a higher service level. At this point, making the appropriate fleet assignment decisions through the network based on the availabilities, seat capacities and demand forecasts becomes more crucial to decrease the operational expenses. It may be preferable to assign a fuel efficient but a smaller aircraft to a certain flight path involving critical passenger connections in albeit of an additional cost of spilled passengers. As it is seen in Table 15, increase in the variability results in more number of spilled passengers as expected. 
Table 14

Comparison of factor effects.

\begin{tabular}{|c|c|c|c|c|c|c|c|c|c|c|c|c|c|}
\hline & & \multicolumn{3}{|c|}{$\begin{array}{l}\text { Fuel \& emis. cost } \\
\text { improvement }\end{array}$} & \multicolumn{3}{|c|}{$\begin{array}{l}\text { Idle cost } \\
\text { improvement }\end{array}$} & \multicolumn{3}{|c|}{$\begin{array}{l}\text { Total cost } \\
\text { improvement }\end{array}$} & \multicolumn{3}{|c|}{$\begin{array}{l}\text { Total impr. } \\
\text { without delay }\end{array}$} \\
\hline & & Min & Avg & $\operatorname{Max}$ & Min & Avg & Max. & Min & Avg & $\operatorname{Max}$ & Min & Avg. & $\operatorname{Max}$ \\
\hline \multirow[t]{2}{*}{$C_{\text {fuel }}(\$ / \mathrm{kg})$} & Low & 2.1 & 3.7 & 4.9 & 69.4 & 72.5 & 78.4 & 23.3 & 25.2 & 28.0 & 20.1 & 23.0 & 26.7 \\
\hline & High & 4.3 & 5.3 & 6.1 & 68.2 & 71.6 & 77.3 & 16.1 & 17.6 & 19.5 & 14.2 & 16.2 & 18.7 \\
\hline \multirow[t]{2}{*}{ Base Spill Cost } & Low & 4.4 & 5.3 & 6.1 & 68.7 & 72.3 & 78.4 & 18.0 & 22.3 & 29.0 & 15.9 & 20.5 & 26.7 \\
\hline & High & 2.1 & 3.7 & 4.8 & 68.2 & 71.9 & 77.6 & 16.1 & 20.6 & 25.9 & 14.2 & 18.7 & 24.7 \\
\hline \multirow[t]{2}{*}{$\beta$} & Low & 2.4 & 4.6 & 6.1 & 68.2 & 72.5 & 78.4 & 16.1 & 21.8 & 28.0 & 15.2 & 20.7 & 26.7 \\
\hline & High & 2.1 & 4.4 & 6.1 & 69.2 & 71.7 & 75.8 & 16.3 & 21.1 & 26.6 & 14.2 & 18.5 & 23.5 \\
\hline
\end{tabular}

Table 15

Factor effects on the percentage of spilled passengers.

\begin{tabular}{lllll}
\hline \multirow{2}{*}{ Factor } & & \multicolumn{3}{l}{$\begin{array}{l}\text { Spilled } \\
\text { passengers }(\%)\end{array}$} \\
\cline { 3 - 5 } & Level & Min & Avg & Max \\
\hline \multirow{2}{*}{$C_{\text {fuel }}(\$ / \mathrm{kg})$} & Low & 0.5 & 1.3 & 2.3 \\
\multirow{3}{*}{ Base Spill Cost } & High & 1.0 & 2.2 & 3.7 \\
& Low & 1.4 & 2.4 & 3.7 \\
$\beta$ & High & 0.5 & 1.0 & 1.5 \\
& Low & 0.5 & 1.7 & 3.4 \\
& High & 0.5 & 1.8 & 3.7 \\
\hline
\end{tabular}

Table 16

CPU time analysis of two-stage algorithm.

\begin{tabular}{lllll}
\hline \multirow{2}{*}{ Factor } & & \multicolumn{3}{c}{ CPU time $(\mathrm{sec})$} \\
\cline { 3 - 5 } \cline { 3 - 5 }$C_{\text {fuel }}(\$ / \mathrm{kg})$ & Level & Min & Avg & Max \\
\multirow{3}{*}{ Base Spill Cost } & 52 & 140 & 269 \\
\multirow{3}{*}{$\beta$} & High & 48 & 113 & 187 \\
& Low & 48 & 137 & 269 \\
& High & 52 & 117 & 214 \\
& Low & 48 & 93 & 158 \\
& High & 80 & 160 & 269 \\
\hline
\end{tabular}

Another measure of interest is the service level of the passengers' connections. For the accuracy of the performance of the generated schedule, the overall desired service level $(\gamma)$ for passenger connections is taken as equal to the overall service level of the published schedule. In the published schedule, for the lower case of $\beta$, the service level is 0.99 , whereas it is 0.96 for the higher case. These values are highly satisfactory to ensure the passenger connections with high probabilities.

Table 16 summarizes the CPU time requirement to achieve these results for the two-stage algorithm. As expected, the value of $\beta$ increases the problem complexity as the increase in the variability. The fuel cost and base spill cost parameters have slightly less effects on the computation time. To solve the larger problem instances with 114 flights, maximum CPU time spent for the twostage algorithm is $120 \mathrm{~s}$, which is still reasonable to generate a robust schedule in real time.

\subsection{Analysis on the schedule with 400 flights}

In our previous results, it can be seen that two-stage algorithm provides very promising results on the schedule with 114 flights operated by 32 different aircraft. In order to demonstrate the actual performance of the algorithmic approach on a more realistic network, we construct another sample schedule which has 400 flights operated by 119 different aircraft, almost quadruple the size of the previous schedule in terms of the number of flights and the number of aircraft. The new flight schedule is also constructed by selecting 400 flights from the work of Arıkan et al. (2016) which was extracted from the database "Airline On-Time Performance
Data" provided by the Bureau of Transportation Statistics of the US Department of Transportation, BTS (2010a).

Although we show that there is no statistically significant randomization effect on the objective function values, for each experimental setting, we still generate five replications. We summarize the results for 40 problems solved by the two-stage algorithm in Table 17.

Table 17 compares the performance of the schedule generated by the two-stage algorithm and the published schedule. It can be seen that results are very promising for relatively larger size of the problems as well. The overall cost saving is approximately $24 \%$, whereas the reduction in idle time cost and fuel and emission costs are around $78 \%$ and $9 \%$, respectively. These are very similar to our previous results. Although the performance of the two-stage algorithm is not affected by the size of the data, the fuel and emission cost savings could be affected by the number of aircraft for each type used in the published schedule.

Moreover, the average CPU time that the two-stage algorithm requires to achieve these results is approximately $6500 \mathrm{~s}$. Maximum amount of time spent is around 13,300 s. It is important to note that these CPU time requirements are reasonable for successful implementation of such a decision making approach in real time.

\section{Conclusions}

We develop a global optimization tool to simultaneously retime the flight departures and assign aircraft types to paths in a given time period. We capture the flight time uncertainty through the chance constraints to ensure passenger connections. In order to increase the passenger connection opportunities, we slightly allow changes in departure times and adjust the speed of the aircraft in response to flight time uncertainty. The crucial contribution of this paper is to consider the fuel burn and $\mathrm{CO}_{2}$ emissions costs associated with adjusting cruise speed to ensure the passenger connections. Therefore, one may prefer to assign a fuel efficient but smaller aircraft to a flight path with critical passenger connections in albeit of an additional cost of spilled passengers. In order to handle the nonlinear costs and chance constraints, we use the conic quadratic programming and obtain the exact solutions as opposed to the approximation methods. We also develop a two-stage algorithm which decomposes the problem into two stages such as aircraft-path assignment and robust airline scheduling, and then solves them sequentially. Computational experiments indicate that the two-stage algorithm performs high quality results in acceptable times.

There are several research directions arising from this work that could be pursued. Great advantages of conic structure of the proposed model enable researchers to integrate more planning stages of airline operations. The current model takes the aircraft paths as input and assigns the aircraft types to these paths. One of the integrations would be to determine the assignment of fleet types to the flight legs without knowing the aircraft path priori. The prob- 
Table 17

Comparison of factor effects of the schedule with 400 Flights.

\begin{tabular}{lllllllllll}
\hline & & \multicolumn{3}{l}{$\begin{array}{l}\text { Fuel \& emis. cost } \\
\text { improvement (\%) }\end{array}$} & \multicolumn{2}{l}{$\begin{array}{l}\text { Idle cost } \\
\text { improvement (\%) }\end{array}$} & \multicolumn{3}{l}{$\begin{array}{l}\text { Total cost } \\
\text { improvement (\%) }\end{array}$} \\
& & Min & Avg & Max & Min & Avg & Max. & Min & Avg & Max \\
\hline \multirow{2}{*}{$C_{\text {fuel }}(\$ / \mathrm{kg})$} & Low & 5.5 & 8.1 & 10.3 & 77.5 & 80.0 & 82.7 & 26.1 & 27.8 & 29.6 \\
& High & 9.0 & 10.2 & 11.3 & 73.7 & 76.5 & 79.6 & 19.0 & 20.6 & 22.2 \\
Base Spill Cost & Low & 9.8 & 10.6 & 11.3 & 75.3 & 78.5 & 81.6 & 21.7 & 25.5 & 29.6 \\
& High & 5.5 & 7.7 & 9.6 & 73.7 & 78.2 & 82.7 & 18.9 & 22.9 & 27.0 \\
& Low & 5.6 & 9.2 & 11.3 & 73.7 & 77.7 & 81.0 & 19.3 & 24.4 & 29.6 \\
& High & 5.5 & 9.1 & 11.2 & 75.2 & 78.9 & 82.7 & 18.9 & 24.0 & 29.2 \\
\hline
\end{tabular}

lem can be further extended to involve the crew assignment decisions as well as the fleet assignment decisions.

One such direction would be to capture the variability in non-cruise times by using stochastic programming. The current method models the variability with chance constraints to ensure the desired service level for passenger connections. The uncertainty would be also handled by a stochastic model where many of potential delay scenarios are analyzed. The effect on delay propagation could be conducted in the enlarged problem involving aircraft, passenger and crew connections through the entire network. On the other hand, considering many scenarios may lead to large decision trees that could requires significant computation time to solve the overall stochastic model.

\section{Appendix A}

\section{A1. Log-Laplace distribution}

The probability density and cumulative distribution functions of Log-Laplace random variable $A_{i}$ with a scale parameter, $e^{\alpha}$ and the tail parameter, $1 / \beta_{i}$ are

$f_{A_{i}}(x)= \begin{cases}\frac{1}{2 \cdot \beta_{i} \cdot x} e^{\frac{(\ln (x)-\alpha)}{\beta_{i}}}, & \text { if } \ln (x)<\alpha \\ \frac{1}{2 \cdot \beta_{i} \cdot x} e^{\frac{-(\ln (x)-\alpha)}{\beta_{i}}}, & \text { if } \ln (x) \geq \alpha\end{cases}$

$F_{A_{i}}(x)= \begin{cases}\frac{1}{2} e^{\frac{(\ln (x)-\alpha)}{\beta_{i}}}, & \text { if } \ln (x)<\alpha \\ 1-\frac{1}{2} e^{\frac{-(\ln (x)-\alpha)}{\beta_{i}}}, & \text { if } \ln (x) \geq \alpha\end{cases}$

The quantile function of the random variable $A_{i}$, which will be used in transformation of the chance constraints into the second order conic inequalities, is given as:

$F_{A_{i}}^{-1}(p)= \begin{cases}(2 p)^{\beta_{i}} \cdot e^{\alpha}, & \text { if } p<\frac{1}{2} \\ \frac{e^{\alpha}}{(2-2 p)^{\beta_{i}}}, & \text { if } p \geq \frac{1}{2}\end{cases}$

\section{A2. Fuel cost function}

To estimate the fuel burn, we used the cruise stage fuel flow model developed by the Base of Aircraft Data (BADA) (EUROCONTROL, 2012). Then, for a given mass of the aircraft and fuel consumption coefficients, fuel burn rate $(\mathrm{kg} / \mathrm{min})$ as a function of the speed $V(\mathrm{~km} / \mathrm{min})$ can be calculated as:

$$
\begin{aligned}
& f_{c r}(V)=\frac{1}{2} \cdot C_{f 1} \cdot C_{f c r} \cdot\left(C_{D 0, C R} \cdot \rho \cdot S \cdot V^{2}+C_{D 0, C R} \cdot \frac{\rho \cdot S}{C_{f 2}} V^{3}\right. \\
& +C_{D 2, C R} \cdot \frac{4 \cdot m^{2} \cdot g_{0}^{2}}{\rho \cdot S \cdot \cos (\phi)^{2} \cdot V^{2}} \\
& \left.\quad+C_{D 2, C R} \cdot \frac{4 \cdot m^{2} \cdot g_{0}^{2}}{C_{f 2} \cdot \rho \cdot S \cdot \cos (\phi)^{2} \cdot V}\right)
\end{aligned}
$$

where aircraft specific fuel consumption coefficients, $C_{f 1}, C_{f 2}, C_{f c r}$, $C_{D 0, C R}$ and $C_{D 2, C R}$, wing surface area, $S$, and mass of the aircraft, $m$, are taken from EUROCONTROL (2012) and listed in Table 5. $\rho$, $g_{0}$ and $\phi$ are the air density $\left(\mathrm{kg} / \mathrm{m}^{3}\right)$ at given altitude, gravitational acceleration $\left(\mathrm{m} / \mathrm{s}^{2}\right)$ and bank angle, respectively. We assume that the distance flown at cruise stage is fixed $d$, and hence the cruise duration is expressed as $d / V$. We can formulate the total fuel consumed $(\mathrm{kg})$ as follows:

$$
\begin{aligned}
F(V)= & \frac{d}{V} \cdot f_{C r}(V) \\
= & \frac{1}{2} \cdot d \cdot C_{f 1} \cdot C_{f c r} \cdot \quad\left(C_{D 0, C R} \cdot \rho \cdot S \cdot V+C_{D 0, C R} \cdot \frac{\rho \cdot S}{C_{f 2}} V^{2}\right. \\
& +C_{D 2, C R} \cdot \frac{4 \cdot m^{2} \cdot g_{0}^{2}}{\rho \cdot S \cdot \cos (\phi)^{2} \cdot V^{3}} \\
& \left.+C_{D 2, C R} \cdot \frac{4 \cdot m^{2} \cdot g_{0}^{2}}{C_{f 2} \cdot \rho \cdot S \cdot \cos (\phi)^{2} \cdot V^{2}}\right)
\end{aligned}
$$

We can rewrite the fuel consumption in terms of the cruise time by replacing $V$ by $\frac{d_{i}}{f_{i}^{t}}$ for each flight $i \in J$ and aircraft $t \in T$. Then, total fuel consumed as a function of cruise time can be expressed as:

$F_{i}^{t}\left(f_{i}^{t}\right)=c_{1}^{i, t} \cdot \frac{1}{f_{i}^{t}}+c_{2}^{i, t} \cdot \frac{1}{\left(f_{i}^{t}\right)^{2}}+c_{3}^{i, t} \cdot\left(f_{i}^{t}\right)^{3}+c_{4}^{i, t} \cdot\left(f_{i}^{t}\right)^{2}$

where

$c_{1}^{i, t}=\frac{1}{2} \cdot C_{f 1}^{t} \cdot C_{f c r}^{t} \cdot C_{D 0, C R}^{t} \cdot \rho \cdot S^{t} \cdot d_{i}^{2}$

$c_{2}^{i, t}=\frac{1}{2} \cdot C_{f 1}^{t} \cdot C_{f c r}^{t} \cdot \frac{C_{D 0, C R}^{t} \cdot \rho \cdot S^{t} \cdot d_{i}^{3}}{C_{f 2}^{t}}$

$c_{3}^{i, t}=\frac{1}{2} \cdot C_{f 1}^{t} \cdot C_{f c r}^{t} \cdot \frac{C_{D 2, C R}^{t} \cdot 4 \cdot m_{t}^{2} \cdot g_{0}^{2}}{\rho \cdot S^{t} \cdot \cos (\phi)^{2} \cdot d_{i}^{2}}$

$c_{4}^{i, t}=\frac{1}{2} \cdot C_{f 1}^{t} \cdot C_{f c r}^{t} \cdot \frac{C_{D 2, C R}^{t} \cdot 4 \cdot m_{t}^{2} \cdot g_{0}^{2}}{C_{f 2}^{t} \cdot \rho \cdot S^{t} \cdot \cos (\phi)^{2} \cdot d_{i}^{2}}$

\section{References}

Ahmadbeygi, S., Cohn, A., Lapp, M., 2010. Decreasing airline delay propagation by re-allocating scheduled slack. IIE Trans. 42, 478-489.

Aktürk, M.S., Atamtürk, A., Gürel, S., 2014. Aircraft rescheduling with cruise speed control. Oper. Res. 62, 829-845.

Arıkan, M., Deshpande, V., 2012. The impact of airline flight schedules on flight delays. Manuf Serv. Oper. Manage 14 (3), 423-440.

Arıkan, M., Deshpande, V., Sohoni, M., 2012. Building reliable air-travel infrastructure using empirical data and stochastic models of airline networks. Oper. Res. 61 (1), 45-64.

Arıkan, U., Gürel, S., Aktürk, M.S., 2016. Integrated aircraft and passenger recovery with cruise time controllability. Ann. Oper. Res. 236 (2), 295-317.

Ben-Tal, A., Nemirovski, A., 2001. Lectures on modern convex optimization: Analysis, algorithms, and engineering applications. SIAM.

Bertsimas, D., Lulli, G., Odoni, A., 2010. An integer optimization approach to large-scale air traffic flow management. Oper. Res. 59, 211-227.

Boeing,. Airplane characteristics for airport planning. http://www.boeing.com/ commercial/airports/plan_manuals.page. Visited June 2014.

BTS, 2010a. Airline on-time performance dataVisited May 2014

BTS, 2010b. T-100 market dataVisited May 2014. 
Burke, E.K., De Causmaecker, P., De Maare, G., Mulder, J., Paelinck, M., Vanden Berghe, G., 2010. A multi-objective approach for robust airline scheduling. Comput. Oper. Res. 37, 822-832.

Chiraphadhanakul, V., Barnhart, C., 2013. Robust flight schedules through slack re-allocation. EURO J.Transp. Logist. 2, 277-306.

Cook, A., Tanner, G., Williams, G., Meise, G., 2009. Dynamic cost indexing-managing airline delay costs. J. Air Transp. Manage. 15, 26-35.

Dunbar, M., Froyland, G., Wu, C.L., 2014. An integrated scenario-based approach for robust aircraft routing, crew pairing and re-timing. Comput. Oper. Res. 45, 68-86.

Duran, A.S., Gürel, S., Aktürk, M.S., 2015. Robust airline scheduling with controllable cruise times and chance constraints. IIE Trans. 47 (1), 64-83.

EUROCONTROL, 2001. Forecasting civil aviation fuel burn and emissions in Europe Technical Report 2001-8. EEC Technical/Scientific, Eurocontrol, Eurocontrol Experimental Centre, B.P. 15, F-91222 Bretigny-sur-Orge, France.

EUROCONTROL, 2012. User manual for the base of aircraft data (BADA) revision 3.10.. Technical Report 12/04/10-45. EEC Technical/Scientific, Eurocontrol, Eurocontrol Experimental Centre, B.P. 15, F-91222 Bretigny-sur-Orge, France.

Günlük, O., Linderoth, J., 2010. Perspective reformulations of mixed integer nonlinear programs with indicator variables. Math Program 124, 183-205.

Hai, J., Barnhart, C., 2013. Robust airline schedule design in a dynamic scheduling environment. Comput. Oper. Res. 40, 831-840.

Hiriart-Urruty, J.B., Lemarećhal, C., 2001. Fundamentals of Convex Analysis. Springer, Berlin.

IATA, 2013. Climate change. http://www.iata.org/policy/environment/Pages/ climate-change.aspx. Visited February 2016.

IATA, 2014. Fuel price analysis. http://www.iata.org/publications/economics/ fuel-monitor/Pages/price-analysis.aspx. Visited May 2014.

ICAO, 2009. ICAO fuel factor. Technical Report. International Civil Aviation Organization (ICAO).

Jarrah, A.I., Goodstein, J., Narasimhan, R., 2000. An efficient airline re-fleeting model for the incremental modification of planned fleet assignments. Transp. Sci. 34 (4), 349-363.

Lan, S., Clarke, J.P., Barnhart, C., 2006. Planning for robust airline operations: optimizing aircraft routings and flight departure times to minimize passenger disruptions. Transp. Sci. 40 (1), 15-28.
Maher, S.J., 2015. A novel passenger recovery approach for the integrated airline recovery problem. Comput. Oper. Res. 57, 123-137.

Marais, K. Waitz IA, 2009. Chapter 14 air transport and the environment. In: Belobaba, P., Odoni, A., Barnhart, C. (Eds.), The Global Airline Industry. Wiley, UK, pp. $405-440$.

Mercier, A., Soumis, F., 2007. An integrated aircraft routing, crew scheduling and flight retiming model. Comput. Oper. Res. 34, 2251-2265.

Papadakos, N., 2009. Integrated airline scheduling. Comput. Oper. Res. 36 (1), 176-195.

Rexing, B., Barnhart, C., Kniker, T., Jarrah, A., Krishnamurthy, N., 2000. Airline fleet assignment with time windows. Transp. Sci. 34 (1), 1-20.

Sherali, H.D., Bae, K.H., Haouari, M., 2013. A benders decomposition approach for an integrated airline schedule design and fleet assignment problem with flight retiming, schedule balance, and demand recapture. Ann. Oper. Res. 210, 213-244.

Sherali, H.D., Bish, E.K., Zhu, X., 2005. Polyhedral analysis and algorithms for a demand-driven refleeting model for aircraft assignment. Transp. Sci. 39 (3), 349-366.

Sherali, H.D., Staats, R.W., Trani, A.A., 2006. An airspace-planning and collaborative decision-making model: part II-cost model, data considerations, and computations. Transp. Sci. 40, 147-164.

Sherali, H.D., Zhu, X., 2008. Two-stage fleet assignment model considering stochastic passenger demands. Oper. Res. 56 (2), 383-399.

Sohoni, M., Lee, Y., Klabjan, D., 2011. Robust airline scheduling under block time uncertainty. Transp. Sci. 45, 451-464.

Tetzloff, I., Crossley, W., 2010. Impact of future generation aircraft on fleet-level environmental emission metrics. AIAA Paper 9205.

T'kindt, V., Billaut, J.C., 2006. Multicriteria scheduling: Theory, models and algorithms, 2 Springer, Berlin.

Weide, O., Ryan, D., Ehrgott, M., 2010. An iterative approach to robust and integrated aircraft routing and crew scheduling. Comput. Oper. Res. 37, 833-844. 